\title{
Treatment of nearly-singular problems with the X-FEM
}

\author{
Grégory Legrain* and Nicolas Moës
}

\author{
*Correspondence: \\ gregory.legrain@ec-nantes.fr \\ LUNAM Université, GeM, UMR CNRS \\ 6183, Ecole Centrale de Nantes, 1 \\ rue de la Noë, 44321 Nantes, France
}

\begin{abstract}
Background: In recent years, lot of research have been conducted on fictitious domain approaches in order to simplify the meshing process for computed aided analysis. The behaviour of such non-conforming methods is studied in the case of the approximation of nearly singular solutions. Such solutions appear when problems involve singularities whose center are located outside (but close) of the domain of interest. These solutions are common in industrial structures that usually involve rounded re-entrant corners.
\end{abstract}

Methods: The performance of the finite element method is evaluated in this context by means of a simple unidimensional example. Both numerical and theoretical estimates are considered in order to assess the behaviour of the numerical approximation. It is demonstrated that despite being regular, the convergence of the approximation can be bounded to an algebraic rate that depends on the solution. Reasons for such behaviour are presented, and two complementary strategies are proposed in order to recover optimal convergence rates. The first strategy is based on a proper enrichment of the approximation thanks to the X-FEM, while the second is based on a proper mesh design that follows a geometric progression. Finally, the proposed strategies are extended and validated in 2D.

Results: The performance of the two strategies is highlighted for both 1D and 2D examples. Both methods allow to recover proper convergence rates (optimal algebraic rate for $h$-convergence, exponential for $p$-convergence) in 1D and $2 \mathrm{D}$.

Conclusions: The proposed strategies allow for a very accurate solution for such solutions. The enrichment strategy is valid for both $h$ and $p$ refinement, whereas the mesh-design strategy is only usable for $p$ refinement. However, such enrichment functions can be tedious to derive.

Keywords: X-FEM; Non-conforming; p-fem; Singularity; Convergence

\section{Background}

Industrial structures usually involve re-entrant corners with possibly small fillets. Accurate stress analysis for such structures requires the proper treatment of these geometrical features. The size of these fillets is highly dependent on the manufacturing process, and depending on the quantity of interest and the size of the fillets with respect to global scale of the structure, it may be neglected in the definition of the mathematical model which is used for the computation. The problem is that such areas with high curvature necessitates the use of very small elements, unless blending mapping [1] or Nurbs-Enhanced FEM $[2,3]$ are used. These very small elements have a high impact of the computational

(c) 2014 Legrain and Moës; licensee Springer. This is an Open Access article distributed under the terms of the Creative Commons Attribution License (http://creativecommons.org/licenses/by/4.0), which permits unrestricted use, distribution, and reproduction in any medium, provided the original work is properly credited. 
cost of the analysis, so that these geometrical features are usually discarded in the analysis and replaced by acute corners. In this case, the numerical solution is not consistent anymore with the mathematical model: the verification of the model is no longer possible, especially if the quantity of interest are related to stresses or strains in the fillet's area. The use of non-conforming approaches such as the X-FEM [4] or fictitious domain [5] can be considered in order to solve this mesh-density issue. Indeed, conforming meshing can be avoided, the price being paid in the integration process. In addition, one has to take care of the correct geometrical description: for example, the use of a level-set for representing the geometry won't allow to obtain an accurate geometry, unless a mesh with a density of the order of the curvature radius is used. Otherwise, one has to consider "sub-grid" level-sets as advocated in [6-8], a fine pixelized representation, as in [9], or the so called Nurbs-Enhanced X-FEM [10]. By means of these strategies, the size of the computational mesh does not have to be related to the size of the geometrical features. The solution being regular, optimal convergence rates are expected. However, albeit being regular, mechanical fields can be very rough in the fillet area. As highlighted in the following, this quasi-singularity prevents an optimal convergence of the solution when using non conforming "engineering" meshes i.e. meshes with a moderate number of elements. The objective of this contribution concerns the quantification of strategies for improving the convergence rate of low and high-order non-conforming finite element methods. Two paths can be followed: (i) using the partition of unity [11] and enrich the finite element approximation with adapted functions; or (ii) using p-fem strategies that are based on non-conforming meshes with proper grading near the singularities [1]. These two strategies are first motivated in a one-dimensional settings, then validated and compared in a 2D setting.

This work is organized as follows: first, mechanical fields near fillets are presented, and their nearly singular behaviour highlighted. In a second part, the eXtended Finite Element Method is introduced together with some recent improvement in the field of high-order approximations. Then, a 1D model problem is introduced in order to highlight the influence of nearly singular fields on the convergence properties of the finite element method. A close study in the error contribution of the elements of the mesh enables us to propose strategies in order to improve the convergence. These strategies are extended in the 2D setting, and validated by means of various numerical examples. Finally, performances of these strategies are compared before concluding.

\section{Near-fillets mechanical fields}

As stated in the introduction, the example of a traction-free blunt re-entrant corner is considered as an example of nearly-singular problem. The geometry of interest is depicted in Figure 1(a). The opening angle of the corner is denoted as $2 \alpha$, the fillet radius as $\rho$ and the local coordinate system as $(r, \theta)$. For comparison purpose, we shall also consider the same problem, but with an acute angle in Figure 1(b). In order to understand the behaviour of the numerical schemes in the presence of blunt corners, the asymptotic mechanical fields near the apex of the corner are presented. The domain is assumed infinite and subjected to a remote uniform tension $\sigma_{0}$. The solution in the case of a sharp notch has been derived by [12]. The closed-form solution in case of a rounded notch has been presented recently by Lazzarin and Tovo [13] and Filippi et al. [14] among 


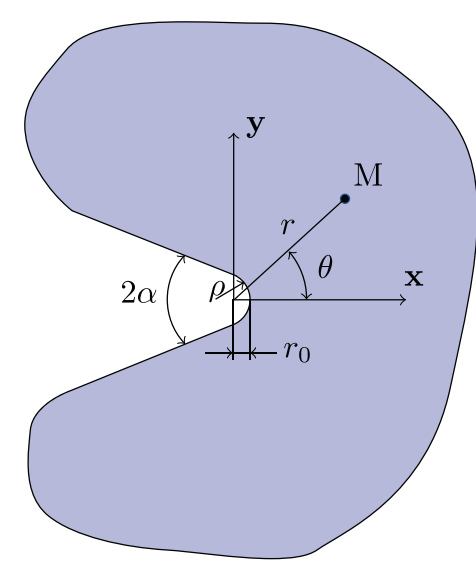

a

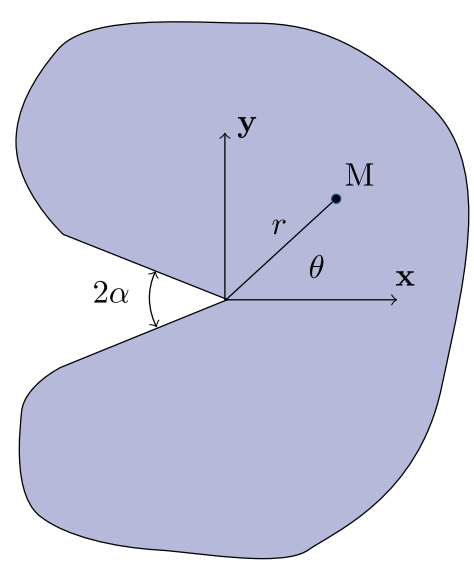

b

Figure 1 Semi-infinite notch: (a) rounded notch; (b) sharp notch.

others. The solution of the problem is obtained by making use of Kolosov-Muskhelishvili's potential. Both solutions are presented, so that their specific features can be highlighted.

\section{Sharp corner}

Following the work of Williams [12], the expression of the stress fields near a traction-free re-entrant corner is written as the sum of two modes that have a different singularity, $\lambda_{1}$ and $\lambda_{2}$. These eigenvalues verify the following equations:

$$
\begin{aligned}
& \sin \left(\lambda_{1} \alpha\right)+\lambda_{1} \sin (\alpha)=0 \\
& \sin \left(\lambda_{2} \alpha\right)-\lambda_{2} \sin (\alpha)=0
\end{aligned}
$$

$\lambda_{1}$ (resp. $\lambda_{2}$ ) is related to a symmetric (resp. anti-symmetric) mode. The expression of the asymptotic stress field is thus [15]:

$$
\sigma_{i j}(r, \theta)=K_{1}^{*} r^{\lambda_{1}-1} f_{i j}^{1}(\alpha, \theta)+K_{2}^{*} r^{\lambda_{2}-1} f_{i j}^{2}(\alpha, \theta)
$$

Factors $K_{i}^{*}$ are called notch stress intensity factors (N-SIFs), and functions $f_{i j}^{k}$ have the following expression:

$$
\left[\begin{array}{l}
f_{r r}^{1} \\
f_{\theta \theta}^{1} \\
f_{r \theta}^{1}
\end{array}\right]=\frac{1}{\lambda_{1}+1+\chi_{b 1}\left(1-\lambda_{1}\right)}\left[\begin{array}{c}
\left(3-\lambda_{1}\right) \cos \left(\left(1-\lambda_{1}\right) \theta\right)-\chi_{b 1}\left(1-\lambda_{1}\right) \cos \left(\left(1+\lambda_{1}\right) \theta\right) \\
\left(\lambda_{1}+1\right) \cos \left(\left(1-\lambda_{1}\right) \theta\right)+\chi_{b 1}\left(1-\lambda_{1}\right) \cos \left(\left(1+\lambda_{1}\right) \theta\right) \\
\left(1-\lambda_{1}\right) \sin \left(\left(1-\lambda_{1}\right) \theta\right)+\chi_{b 1}\left(1-\lambda_{1}\right) \sin \left(\left(1+\lambda_{1}\right) \theta\right)
\end{array}\right]
$$

and:

$$
\left[\begin{array}{l}
f_{r r}^{2} \\
f_{\theta \theta}^{2} \\
f_{r \theta}^{2}
\end{array}\right]=\frac{1}{\lambda_{2}+1+\chi_{b 2}\left(1-\lambda_{2}\right)}\left[\begin{array}{c}
\left(3-\lambda_{2}\right) \sin \left(\left(1-\lambda_{2}\right) \theta\right)-\chi_{b 1}\left(1-\lambda_{2}\right) \sin \left(\left(1+\lambda_{2}\right) \theta\right) \\
\left(\lambda_{2}+1\right) \sin \left(\left(1-\lambda_{2}\right) \theta\right)+\chi_{b 1}\left(1-\lambda_{2}\right) \sin \left(\left(1+\lambda_{2}\right) \theta\right) \\
\left(1-\lambda_{2}\right) \cos \left(\left(1-\lambda_{2}\right) \theta\right)+\chi_{b 1}\left(1-\lambda_{2}\right) \cos \left(\left(1+\lambda_{2}\right) \theta\right)
\end{array}\right]
$$


where (and using the notation $\alpha=q \pi$ ):

$$
\begin{aligned}
& \chi_{b 1}=-\frac{\sin \left(\left(1-\lambda_{1}\right) q \pi / 2\right)}{\sin \left(\left(1+\lambda_{1}\right) q \pi / 2\right)} \\
& \chi_{b 2}=-\frac{\sin \left(\left(1-\lambda_{2}\right) q \pi / 2\right)}{\sin \left(\left(1+\lambda_{2}\right) q \pi / 2\right)}
\end{aligned}
$$

It can be seen that both modes produce singular stresses at the apex of the corner when $\lambda_{i}<1$, which causes a loss of convergence for finite elements, as discussed in sections 'Discussion' and 'Convergence for nearly singular problems'. In particular, for $2 \alpha=\pi / 2$, one finds $\lambda_{1}=0.5448$ and $\lambda_{2}=0.9085$ : Mode I is more singular than mode II.

\section{Rounded corner}

The determination of the asymptotic stresses near a rounded corner has been studied by Creager and Paris [16], Glinka [17], Lazzarin and Tovo [13], and improved recently by Filippi et al. [14]. The two last contributions are based on the use of KolosovMuskhelishvili's potentials together with an auxiliary system of curved coordinates that mimics the rounded corner's geometry. This conformal mapping approximation of the rounded notch has an hyperbolic shape which will have to be taken into account in the numerical examples (see Figure 2).

Following [14], the stress distribution near the rounded notch is seen to be:

$$
\sigma_{i j}(r, \theta)=K_{1}^{*} r^{\lambda_{1}-1}\left[f_{i j}^{1}(\alpha, \theta)+g_{i j}^{1}(r, \alpha, \theta)\right]+K_{2}^{*} r^{\lambda_{2}-1}\left[f_{i j}^{2}(\alpha, \theta)+g_{i j}^{2}(r, \alpha, \theta)\right]
$$

It is important to note that functions $f_{i j}^{k}$ are the same as in the case of the sharp corner. Nevertheless, the resulting stress field is not singular as the origin of the frame is out of the domain (see Figure 1(a)). The expression of functions $g_{i j}^{k}$ are the following:

$$
\begin{aligned}
{\left[\begin{array}{l}
g_{r r}^{1} \\
g_{\theta \theta}^{1} \\
g_{r \theta}^{1}
\end{array}\right]=} & \frac{q\left(\frac{r}{r_{0}}\right)^{\mu_{1}-\lambda_{1}}}{4(q-1)\left[\lambda_{1}+1+\chi_{b 1}\left(1-\lambda_{1}\right)\right]} \\
& \times\left[\begin{array}{c}
\chi_{d 1}\left[\left(3-\mu_{1}\right) \cos \left(\left(1-\mu_{1}\right) \theta\right)\right]-\chi_{c 1} \cos \left(\left(1+\mu_{1}\right) \theta\right) \\
\chi_{d 1}\left[\left(\mu_{1}+1\right) \cos \left(\left(1-\mu_{1}\right) \theta\right)\right]+\chi_{c 1} \cos \left(\left(1+\mu_{1}\right) \theta\right) \\
\chi_{d 1}\left[\left(1-\mu_{1}\right) \sin \left(\left(1-\mu_{1}\right) \theta\right)\right]+\chi_{c 1} \sin \left(\left(1+\mu_{1}\right) \theta\right)
\end{array}\right]
\end{aligned}
$$
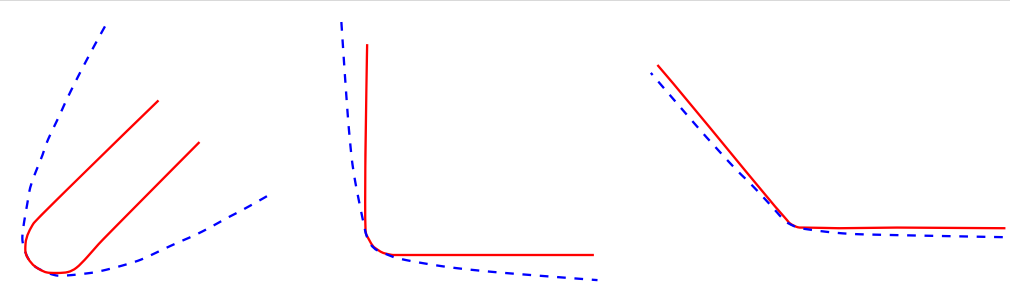

Figure $\mathbf{2}$ Analytical (dashed blue) and real (solid red) geometries for various opening angles. It can be noted that the larger the angle, the better the approximation of the real geometry. 
and:

$$
\begin{aligned}
{\left[\begin{array}{c}
g_{r r}^{2} \\
g_{\theta \theta}^{2} \\
g_{r \theta}^{2}
\end{array}\right]=} & \frac{q\left(\frac{r}{r_{0}}\right)^{\mu_{2}-\lambda_{2}}}{4(q-1)\left[\lambda_{2}+1+\chi_{b 2}\left(1-\lambda_{2}\right)\right]} \\
\times & {\left[\begin{array}{c}
\chi_{d 2}\left[\left(3-\mu_{2}\right) \sin \left(\left(1-\mu_{2}\right) \theta\right)\right]-\chi_{c 2} \sin \left(\left(1+\mu_{2}\right) \theta\right) \\
\chi_{d 2}\left[\left(\mu_{2}+1\right) \sin \left(\left(1-\mu_{2}\right) \theta\right)\right]+\chi_{c 2} \sin \left(\left(1+\mu_{2}\right) \theta\right) \\
\chi_{d 2}\left[\left(1-\mu_{2}\right) \cos \left(\left(1-\mu_{2}\right) \theta\right)\right]+\chi_{c 2} \cos \left(\left(1+\mu_{2}\right) \theta\right)
\end{array}\right] }
\end{aligned}
$$

Factors $\chi_{c 1}, \chi_{c 2}, \chi_{d 1}$ and $\chi_{d 2}$ are given in [14], and $\mu_{1}$ and $\mu_{2}$ are solutions of the following non-linear equations:

$$
\begin{aligned}
& \left\{\frac{1-q\left(1+\mu_{1}\right)}{q}\left[3-\lambda_{1}-\chi_{b 1}\left(1-\lambda_{1}\right)\right]-\epsilon_{1}\right\}\left(1+\mu_{1}\right) \cos \left[\left(1-\mu_{1}\right) q \frac{\pi}{2}\right] \\
& \quad+\left\{\left[\left(1-\mu_{1}\right)^{2}-\frac{1+\mu_{1}}{q}\right]\left[3-\lambda_{1}-\chi_{b 1}\left(1-\lambda_{1}\right)\right]-\left(3-\mu_{1}\right) \epsilon_{1}\right\} \cos \left[\left(1+\mu_{1}\right) q \frac{\pi}{2}\right]=0
\end{aligned}
$$

and:

$$
\begin{aligned}
& \left\{\left[\frac{q\left(1+\mu_{2}\right)-2}{q}\right]\left[\lambda_{2}-1-\chi_{b 2}\left(1+\lambda_{2}\right)\right]-\epsilon_{2}\right\}\left(1-\mu_{2}\right) \cos \left[q \frac{\pi}{2}\left(1-\mu_{2}\right)\right] \\
& \quad+\left\{\left(\mu_{2}-1\right)\left[\frac{q\left(\mu_{2}-3\right)-2}{q}\right]\left[\lambda_{2}-1-\chi_{b 2}\left(1+\lambda_{2}\right)\right]+\left(1-\mu_{2}\right) \epsilon_{2}\right\} \cos \left[q \frac{\pi}{2}\left(1+\mu_{2}\right)\right]
\end{aligned}
$$

where $\epsilon_{1}$ and $\epsilon_{2}$ are also given in [14]. All the coefficients presented before can be evaluated from the knowledge of the geometry of the rounded corner (i.e. its opening angle $2 \alpha$ and its radius of curvature $\rho$ ). Finally, it is possible to obtain the displacement field associated with this asymptotic stress field, by means of the constitutive law and proper integration.

The displacement field has the following form:

$$
\begin{aligned}
& u_{r}(r, \theta)=u_{r}^{s, 1}(r, \theta)+u_{r}^{s, 2}(r, \theta)+u_{r}^{b, 1}(r, \theta)+u_{r}^{b, 2}(r, \theta) \\
& u_{\theta}(r, \theta)=u_{\theta}^{s, 1}(r, \theta)+u_{\theta}^{s, 2}(r, \theta)+u_{\theta}^{b, 1}(r, \theta)+u_{\theta}^{b, 2}(r, \theta)
\end{aligned}
$$

The explicit expression of functions $u_{r}^{s, i}$ and $u_{r}^{b, i}$ are given in Appendix A, and a sketch of the first mode displacement and strain are presented in Figures 3 and 4.

\section{Discussion}

In order to discuss the expected behaviour of the asymptotic solution derived in the previous section, let us consider the terminology coined in [1]. This classification, based on 


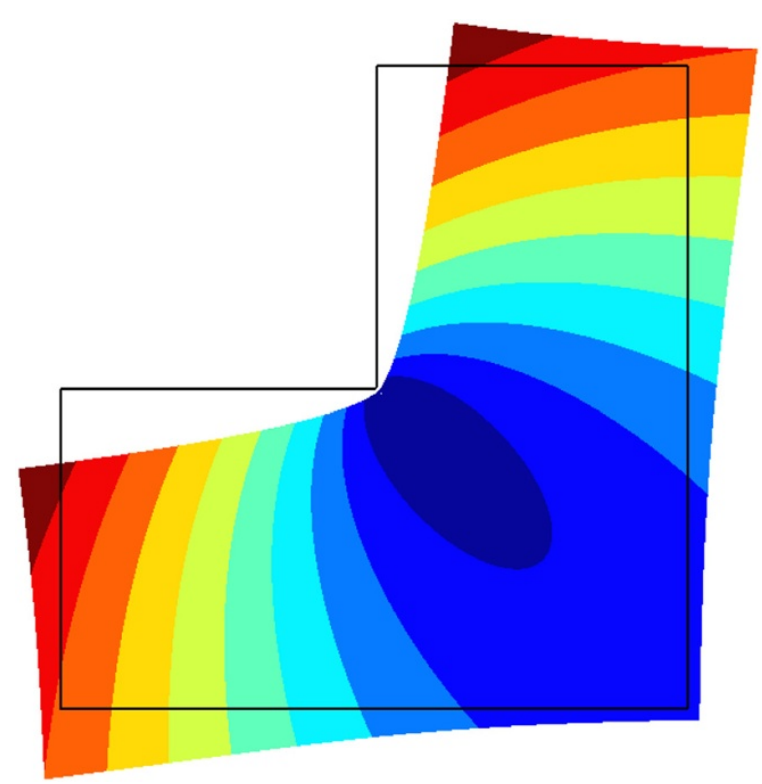

Figure 3 Displacement field associated to a blunt corner of radius 0.00625 subjected to a mode I loading.

the features of the analytical solution of a problem, enables to predict the behaviour of the finite element method. Solutions can be separated in three classes:

Category A If the solution is analytic everywhere in the domain (including boundaries);

Category B If the solution is analytic everywhere, except at a finite number of singular points (and edges in 3D);

Category $\mathrm{C}$ If the solution does not belong to the previous categories (material interfaces for example);

Practical problems usually belong to category B. Note however that the solution is not necessarily singular near singular points: it depends on the eigenvalues of the expansion of the solution. If the eigenvalues are strictly smaller than one, then the solution is

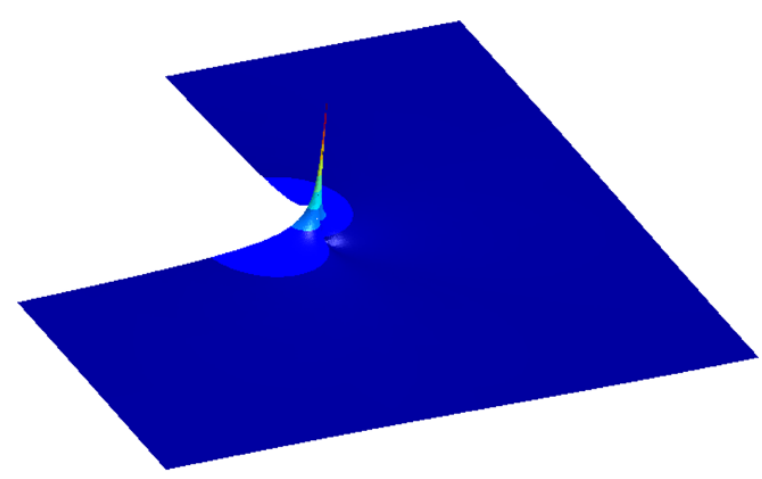

Figure 4 Strain field (Von-Mises norm) associated to a blunt corner of radius 0.00625 subjected to a mode I loading. 
singular and the problem is said strongly in category B. Otherwise, it is qualified as weakly in category B.

As stated in section 'Sharp corner', the stress field associated with the sharp corner eqn. (3) is singular and problems involving these geometrical features belong strongly to category B. In this case, the convergence of the finite element method is bounded by the order of the singularity of the solution i.e. $\min \left(\lambda_{1}, \lambda_{2}\right)$. Note however that if $2 \alpha \geq \pi$, then $\lambda_{i} \geq 1$, and the problem becomes regular (weakly in category $\mathrm{B}$ ). The convergence is thus bounded by the polynomial order of the approximation (for $h$ finite elements). On the contrary, the stress field related to the rounded corner eqn. (8) is regular, although it can be very rough if the radius of curvature $\rho$ is small. The problem is then always weakly in category $\mathrm{B}$, but if $\rho$ is small then it tends to be strongly in category $\mathrm{B}$. As the solution is regular, one would expect $h$ convergence rates associated with the order $p$ of the polynomial approximation (i.e. in $\mathcal{O}\left(h^{p}\right)$ in the energy norm). This is the case asymptotically, but not necessary for "engineering meshes" (meshes with a moderate number of elements), as illustrated in section '1D model problem'.

\section{The eXtended finite element method}

In the following, the eXtended Finite Element Method (X-FEM) will be used for the computations. The X-FEM [4] is an extension of the finite element method (FEM) that was developed from the need to improve the FEM approach for problems with complex geometrical features (cracks [4], material interfaces [18], free surfaces [18,19]). In contrast to classical finite elements, the X-FEM does not require the mesh to conform the geometry. Instead, a regular mesh is constructed for the domain of interest and the presence of internal boundaries is taken into account in the formulation of the finite elements at the corresponding locations by means of the partition of unity method [11]. The X-FEM approximation of the displacement field, $\mathbf{u}$, over an element $\Omega_{e}$ is given by:

$$
\left.\mathbf{u}(\mathbf{x})\right|_{\Omega_{e}}=\sum_{\alpha=1}^{n} \mathbf{N}^{\alpha}\left(u^{\alpha}+\sum_{\beta=1}^{n_{e}} a_{\beta}^{\alpha} \varphi_{\beta}(\mathbf{x})\right)
$$

where the approximation can be divided into a classical one that depends only on the vectorial shape functions $\mathbf{N}^{\alpha}(x)^{\mathrm{a}}$ and classical degrees of freedom (dofs in the following) $u^{\alpha}$, and an enriched one that depends on enrichment functions $\varphi_{\beta}(x)$ and enriched dofs $a_{\beta}^{\alpha}$. Those functions prevent poor rates of convergence due to the non-conformity of the approximation or the singularity of the solution. The additional degrees of freedom are only added at the nodes whose support is split by the interface, which means that typically only a few of them are added. More precisely, if an element is fully enriched, then this number of enriched dofs is equal to $n \times n_{e}$. On the contrary, no enrichment is used in the case of a non-conforming approximation: the weak form is just integrated selectively in the domain. A level-set representation of the geometry is typically used: in this case, the level-set is interpolated on the approximation mesh. This couples the geometrical representation to the approximation [20], and prevents the use of higher order approximations due to an insufficient geometrical accuracy. A so-called sub-grid level-set approach has been proposed in [6,7] in order to uncouple geometry and approximation, and thus allow the use of high-order approximations. Alternatively, the use of the so-called Nurbs-Enhanced X-FEM [10] allows to consider the exact geometrical representation independently of the approximation mesh, see Figure 5 for a comparison of both 
approaches. In this case, geometries such as the one depicted in Figure 6 could be represented using a mesh whose characteristic length is large with respect to the geometrical details. However, the following question arise: what is the influence of the size mismatch between the mesh and the geometrical details on the convergence of the finite element approximation?

In this contribution, both low and high-order X-FEM will be considered. The latter case makes sense, as one can deal with meshes composed of big elements with simple shape. In this case, the enrichment scheme presented in (14) can lead to conditioning issues when a so-called geometrical enrichment is used [21]. Geometrical enrichment states that the size of the enriched region remains unchanged during refinement (it is also called "fixed area" enrichment in [22]). It is not related to the concept of "geometric mesh" which is commonly used in the $p$-fem community. In order to improve this issue, several strategies have been proposed [21-24]. In this contribution, the strategy proposed by Duarte et al. [25] and further studied in Chevaugeon et al. [26] is considered. It consists in using a vectorial enrichment, rather than a scalar one as in eqn. (14):

$$
\left.\mathbf{u}(\mathbf{x})\right|_{\Omega_{e}}=\sum_{\alpha=1}^{n} \mathbf{N}^{\alpha} u^{\alpha}+\sum_{\alpha=1}^{\tilde{n}} \tilde{N}^{\alpha}\left(\sum_{\beta=1}^{n_{e}} a_{\beta}^{\alpha} \boldsymbol{\varphi}_{\beta}(\mathbf{x})\right)
$$

in this expression, the first term corresponds to the classical finite element approximation while the second one corresponds to the enrichment. It involves $\tilde{N}^{\alpha}$, the scalar shape

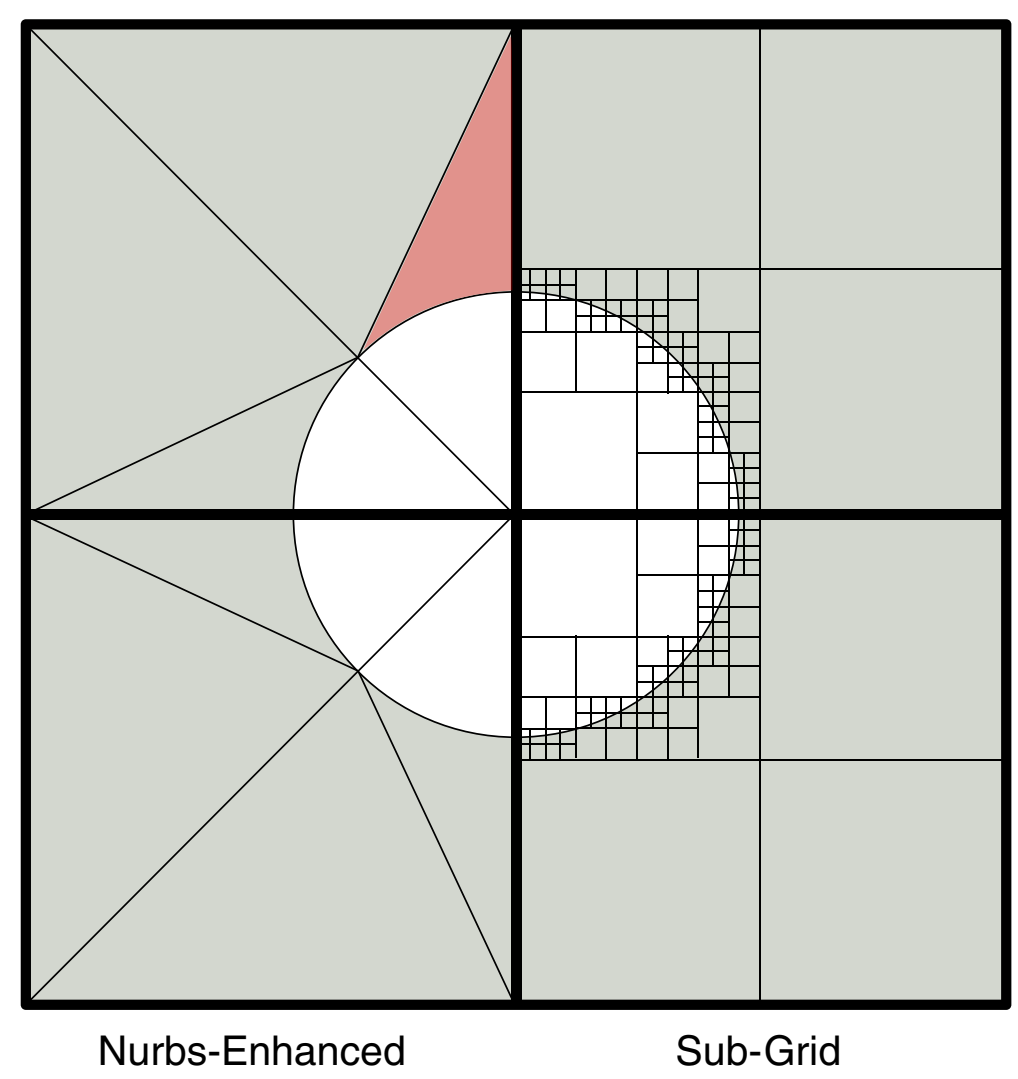

Figure 5 Left: Nurbs-Enhanced X-FEM (in red, a typical integration cell); Right: Sub-Grid level-set. Thick lines indicate elements boundaries, and thin lines integration cells boundaries. 


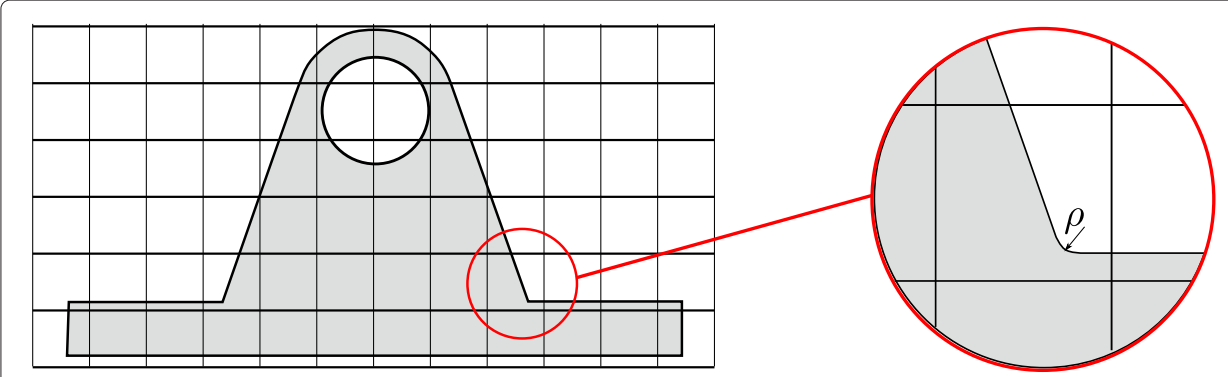

Figure 6 Geometrical details in an element.

function associated with the partition of unity, $a_{\beta}^{\alpha}$ the scalar enriched dof and $\boldsymbol{\varphi}_{\beta}(\mathbf{x})$ the $\beta^{\text {est }}$ vectorial enrichment function. Note that the number of vectorial shape functions $n$ remains unchanged with respect to (14), and that the number of scalar shape functions $\tilde{n}$ is smaller than $n$. More precisely, in the case where $\tilde{N}$ and $\mathbf{N}$ share the same polynomial order, we have $\tilde{n}=\frac{n}{d}$ with $d$ the spatial dimension of the problem. It reflects the different nature of these shape functions (vectorial and scalar). This difference has also an influence on the number of enriched dofs: it is reduced by a factor $d$ if (15) is used $\left(\tilde{n} \times n_{e}\right.$ rather than $\left.n \times n_{e}\right)$.

In [26], the resulting conditioning number evolution was shown to increase in $O\left(1 / h^{2}\right)$ for a model problem, which is the same rate as classical linear finite elements. This improvement in the conditioning number is of great interest in practice, as is allows to use the so-called geometrical enrichment which has been proved to be optimal in term of convergence. This aspect becomes fundamental when high-order shape functions are used, as the conditioning number increases with the polynomial order.

\section{Methods}

\section{D model problem}

The behaviour of the finite element approximation is studied on a simple 1D model problem which is representative of the solution near the fillet:

$$
\begin{gathered}
\left.\frac{d^{2} u}{d x^{2}}+f=0 \quad x \in\right] 0,1[ \\
u(0)=u(1)=0
\end{gathered}
$$

$f$ is chosen such that the exact solution is:

$$
u_{0}(x)=x^{\alpha}-x
$$

for $\alpha>1 / 2$. One can see that this solution is singular, and that the center of the singularity is for $x=0$. Such a solution can be compared to typical 2D solutions near re-entrant corners: $u=r^{\beta} \phi(\theta)$ for $\alpha=\beta+1 / 2$. The problem is solved using $h$ and $p$ finite element approximations, with both homogeneous or geometric meshes [27]. The finite element shape functions are based on integrated Legendre polynomials, as presented in [1]. The problem is solved for $x \in[\varepsilon, 1[, \varepsilon \in[0,1[$. Remark that a conforming mesh is used for this simple problem: the conclusions can be extended to the non-conforming case. We first consider the case where $\varepsilon=0$ : the singularity emanates from the boundary of the 
domain. In the case of a quasi-uniform mesh, the estimates given in [27] states that the energy norm of the error evolves as:

$$
\|e\|_{E} \leq k N^{-\beta}
$$

with $\beta=\min (p, \alpha-1 / 2)$ for $h$-refinement, and $\beta=2 \alpha-1$ for $p$-refinement. Note that in both cases the convergence is algebraic, with an order which depends on the singularity of the solution. In the case where the singularity lies out of the domain, the convergence remains algebraic for $h$-refinement, whereas it becomes exponential for $p$-refinement [28]:

$$
\|e\|_{E} \leq k \exp ^{-\gamma N^{\theta}}
$$

In this expression, $k, \gamma$ and $\theta$ are positive constants that depend on the exact solution.

\section{Convergence for nearly singular problems}

Equations (19) and (20) are checked on the model problem (16) with $\alpha=0.55$, and for $\varepsilon=10^{-k}, k \in[1,2,3,4,5]$. Both $h$ and $p$-convergence are considered, with a regular nodal distribution. Note that all the figures in this section represent absolute errors. The results are presented in Figures 7 and 8: it can be seen that $\varepsilon$ has a visible effect on the behaviour of the two approaches. Large $\varepsilon$ values correspond to very smooth solutions in the domain, so that estimates (19) with $\beta=p$ and (20) are verified. On the contrary, small values for $\varepsilon$ correspond to nearly-singular solutions in spite of the absence of any singularity in the domain and on its boundary. The convergence tends to be algebraic with a rate that depends on the singularity ( $\alpha-1 / 2$ for $h$-convergence, and $2 \alpha-1$ for $p$-convergence). In particular, two regimes can be observed in Figure 7: for low $h$ the convergence is driven by the singularity $(\beta=\alpha-0.5)$, whereas at some point the asymptotic $\beta=p$ convergence is observed. The smaller $\varepsilon$, the later this asymptotic convergence is recovered. Note that

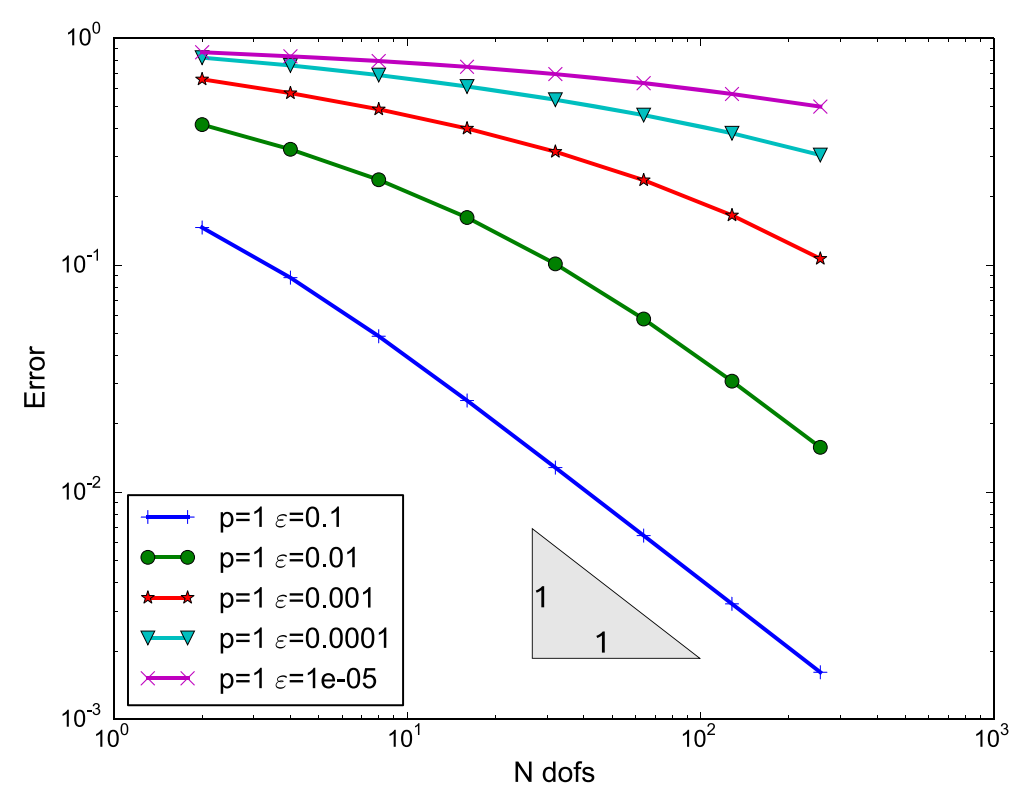

Figure 7 Influence of the distance to the singularity, $h$-convergence. 


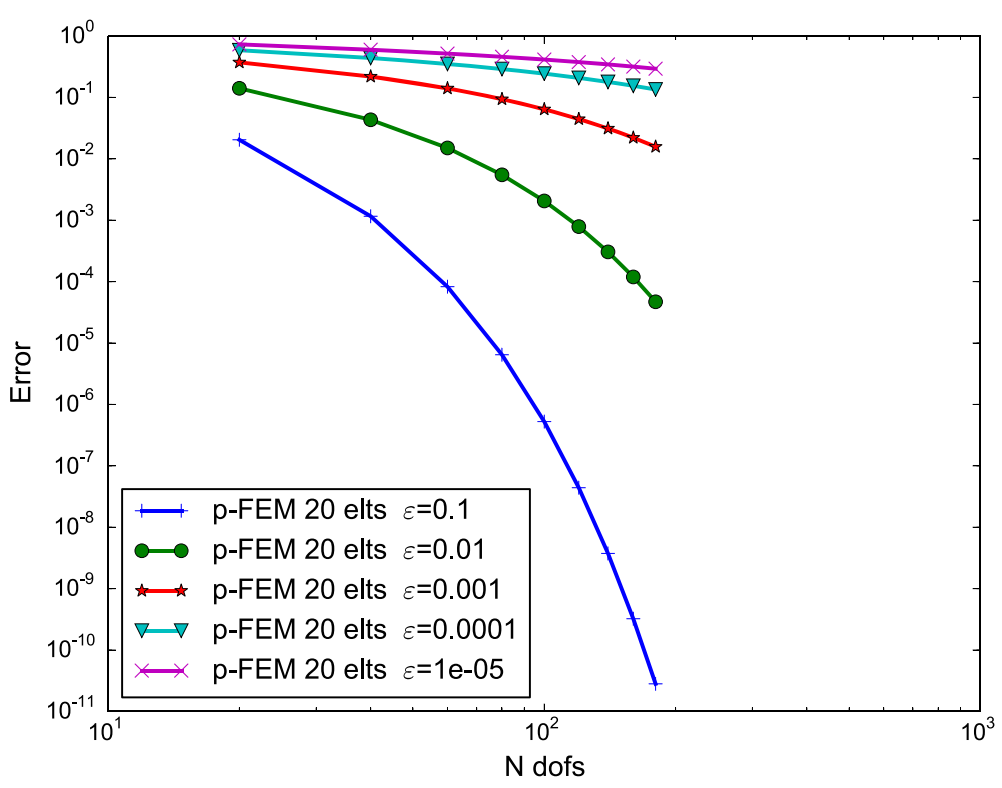

Figure 8 Influence of the distance to the singularity, $p$-convergence.

this regular convergences can always be obtained, but not necessarily for "engineering" meshes (i.e. with moderate element size).

The reason for this loss of convergence is now discussed more precisely in the $p$-Fem case: Consider the contribution of the first four elements to the global error which is presented in Figure 9(a) (for $\alpha=0.55, \varepsilon=10^{-5}$ and 20 elements). It can be seen that only the first element has an algebraic convergence, whereas the remaining elements converge exponentially. This behaviour is typical of the singular case, where this phenomenon is well known. For bigger $\varepsilon$, an exponential convergence is obtained for all the elements (see Figure 9(b)).

Following [27] (in the case where $\varepsilon=0$ ), the contribution $\eta_{1}$ of the first element (that touches the center of the singularity) to the error writes:

$$
\eta_{1} \simeq C_{0}(\alpha) \frac{h_{1}^{\alpha-1 / 2}}{p^{2 \alpha-1}}\left(1+O\left(\frac{1}{p}\right)\right) \quad(p \rightarrow \infty)
$$

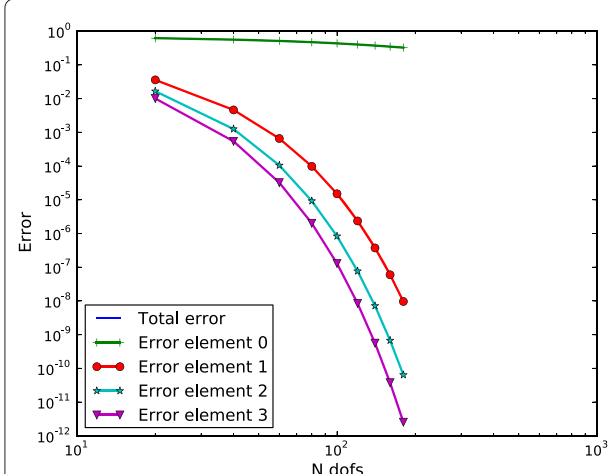

a

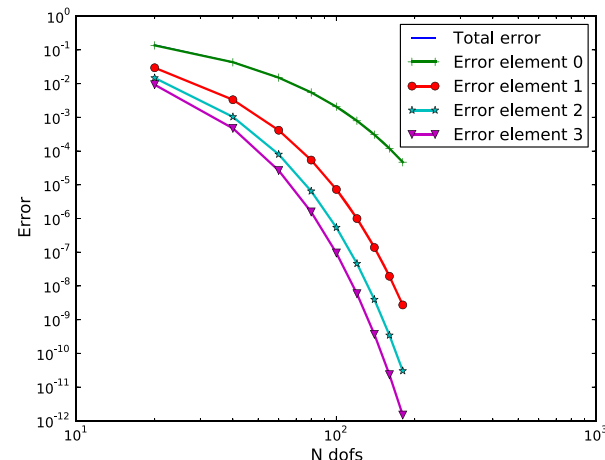

b

Figure 9 Contribution of the first elements to the global error: (a) $\varepsilon=10^{-5}$; (b) $\varepsilon=10^{-2}$. 
Where $h_{1}$ is the length of the element, and $C_{0}(\alpha)$ is a constant. This shows that the error decrease in the first element is algebraic. On the contrary, the estimate for the remaining elements is:

$$
\begin{aligned}
\eta_{i, i>1} & \simeq C_{1}(\alpha) h_{i}^{\alpha-1 / 2}\left(\frac{1-r_{i}^{2}}{2 r_{i}}\right)^{\alpha-1} \frac{r_{i}^{p}}{p^{\alpha}}\left(1+O\left(\frac{1}{p^{\sigma}}\right)\right) \\
r_{i} & =\frac{\sqrt{x_{i}}-\sqrt{x_{i-1}}}{\sqrt{x_{i}}+\sqrt{x_{i+1}}}
\end{aligned}
$$

Where $h_{i}$ is the length of the element, $C_{1}(\alpha)$ is a constant, and $\sigma>0$. This equations states that the convergence is exponential in all the elements but the first one. This is why exponential convergence should be expected in the nearly-singular case, as all the elements should follow estimate (22) (none of them touches the center of the singularity). Although, Figure 9(a) seems to contradict eqn. (22), this is not the case. Indeed, eqn. (22) holds only if $0<r_{i}^{2}<1-1 / p$, which is not the case for Figure 9(a), as $r^{2}$ ranges from 0.999 to $6.610^{-4}$ (see Table 1). It can be seen that only the first element has a $r^{2}$ greater than $1-1 / p \forall p$, hence the algebraic convergence. When $1-1 / p<r_{i}^{2}<1$, following [28], the estimates becomes:

$$
E\left(I_{i}\right) \simeq h_{i}^{\alpha-1 / 2} \frac{r_{i}^{p+1-\alpha}}{p^{\alpha-1 / 2}}\left(1 / p^{\alpha-1 / 2}+\left(1-r_{i}^{2}\right)^{\alpha-1 / 2}\right)
$$

When $r_{i}^{2}$ is close to 1 , one obtain the same estimate as eqn. (21), which is consistent with the numerical results (Figure $9(\mathrm{a}))^{\mathrm{b}}$.

\section{Strategies for recovering optimal convergence}

Two strategies are proposed in order to recover a proper convergence in the case of nearly-singular problems. The first one is based on an enrichment of the approximation, using the Partition of Unity method [11], see eqn. (14). The second one is based on a proper mesh design, which is close to the approaches that are classically used in the context of $p$-Fem.

\section{Enrichment of the approximation}

The idea consists in the enrichment of the approximation in order to capture the steep gradients of the exact solution. The enrichment function considered is $x^{\alpha}$ as only this term is singular in (18), and a "geometrical" enrichment strategy is considered, as it has been shown in practice that it was leading to better convergence properties [21,22]. Such an approach can be used for both $h$ and $p$ Fem.

Table 1 Evolution of $r^{2}$ for the elements of a regular mesh $\left(\varepsilon=10^{-5}, 20\right.$ elements)

\begin{tabular}{cc}
\hline Element & $r^{\mathbf{2}}$ \\
\hline 1 & $9.9910^{-1}$ \\
2 & $1.1110^{-1}$ \\
3 & $4.0010^{-2}$ \\
4 & $2.0410^{-2}$ \\
20 & $6.5710^{-4}$ \\
\hline
\end{tabular}




\section{Suitable mesh design}

A second possibility, based on the construction of a suitable mesh is investigated in the case when $p$-refinement is considered. It has been highlighted in the previous section that in the case of $p$-Fem, the contribution of the first element was preventing any exponential convergence of the approximation. A sufficient condition for recovering the exponential convergence consists in ensuring that this first element converges exponentially. The objective is thus to prescribe $r^{2}<1-\frac{1}{p}$ in the first element. In the context of $p$ refinement, we have chosen to verify this condition for small orders, i.e. $p=2$. So, if $r^{2}<1 / 2$ exponential convergence is expected for all the elements and for any polynomial order greater than two. First, consider the case of a regular mesh depicted in Figure 10. We are interested in the first element which is highlighted in red. This element is located at a distance $\varepsilon$ from the center of the singularity. For this first element, condition $r^{2}<1 / 2$ can be written as:

$$
\left(\frac{\sqrt{\varepsilon+h}-\sqrt{\varepsilon}}{\sqrt{\varepsilon+h}+\sqrt{\varepsilon}}\right)^{2} \leq \frac{1}{2}
$$

Solving this equation for $h$ gives the maximum length of the first element allowing for an exponential convergence:

$$
\begin{aligned}
& h \in[0,4(3 \sqrt{2}+4) \varepsilon] \\
& h \in[0,33 \varepsilon]
\end{aligned}
$$

Equation (25) states that in the case of a quasi-uniform mesh, the length of the first elements must have the same order of magnitude as $\varepsilon$. This condition is very restrictive in practice, as a quasi-uniform mesh of this type is unusable for real problems. Note that the numerical results from Figure 8 are consistent with this estimate, as exponential convergence is noticeable for $\varepsilon<10^{-3}$ which is close to $h / 33 \simeq 1.610^{-3}$.

We now consider the use of a geometrical mesh. Indeed, it has been shown in the case of singular problems [1] that the use of a finite element mesh with geometrical progression of power 0.15 near the center of the singularity could lead to exponential rates of convergence for both $p$ and $h-p$ fem (only in the pre-asymptotic range for the former case, while this rate can be maintained asymptotically in the latter). It is interesting to note that the geometric progression is independent of the order of the singularity. In this case, factor $r$ is seen to be constant, and the condition $r^{2}<1 / 2$ becomes:

$$
\left(\frac{1-\sqrt{q}}{1+\sqrt{q}}\right)^{2} \leq \frac{1}{2}
$$

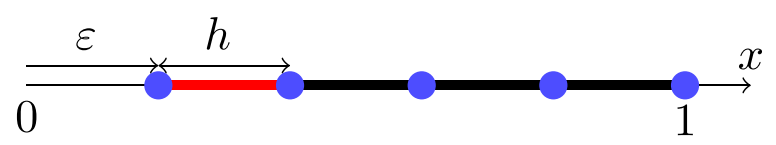

Figure 10 Regular mesh with singularity out of the domain $(x=0)$. In red, the element of interest. 
Solving this equation for $0<q<1$ gives the range of geometrical progression that allows for an exponential convergence:

$$
\begin{aligned}
& q \in\left[\frac{1}{17+12 \sqrt{2}}, 1\right] \\
& q \in[0.029,1]
\end{aligned}
$$

From this study one can see that in practice (i.e. $q \geq 0.15$ ), the exponential convergence is ensured no matter the value of the geometrical progression.

\section{Results and discussion}

\section{D Numerical examples}

The two strategies presented above are now appraised considering $\alpha=0.55$ and $\varepsilon=10^{-5}$ (i.e. for the most unfavourable case). The energy norm of the error is monitored with respect to the number of degrees of freedom.

\section{h-convergence}

Only the enrichment strategy is considered in this case. The length of the enriched zone is set to 0.25 , and $h$ varies from 0.0625 to 0.00390625 . The selection of the enriched nodes upon mesh refinement is illustrated is Figure 11, where the enriched nodes are depicted as squares. The results are presented in Figure 12, and it can be seen that (regular) optimal order of convergence are obtained for linear, quadratic and cubic approximations. Note the small loss in the convergence for P2 and small $h$ which stems from the accuracy of the integration of the weak formulation.

\section{p-convergence}

In this section, both enrichment and mesh-based strategies are considered. In the case of the enrichment strategy, a four elements mesh is considered and only the first one is enriched (note that in this case, the enrichment strategy behaves like a "geometrical" enrichment). The polynomial order ranges from 1 to 10, and the evolution of the error in the energy norm is depicted in Figure 13. One can see that the exponential rate of convergence is recovered. This exponential convergence makes it possible to obtain a $10^{-8}$ absolute error level with ten times less dofs, compared to enriched h-convergence.

Finally, the use of a geometrical mesh is considered. Various geometrical progressions are used, ranging from 0.0032 to 0.24 : a typical mesh with four elements ( 0.0562 progression) is depicted in Figure 14 to illustrate the strong grading near the singularity. It can be

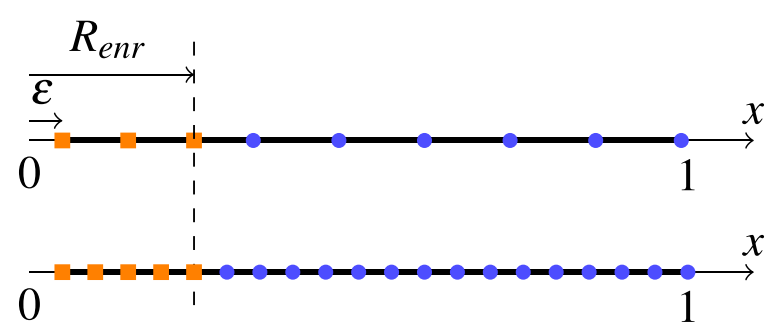

Figure 11 "Geometrical" enrichment strategy for two mesh size: $\boldsymbol{R}_{\text {enr }}$ corresponds to the size of the enriched zone, and square marks represent enriched nodes. 


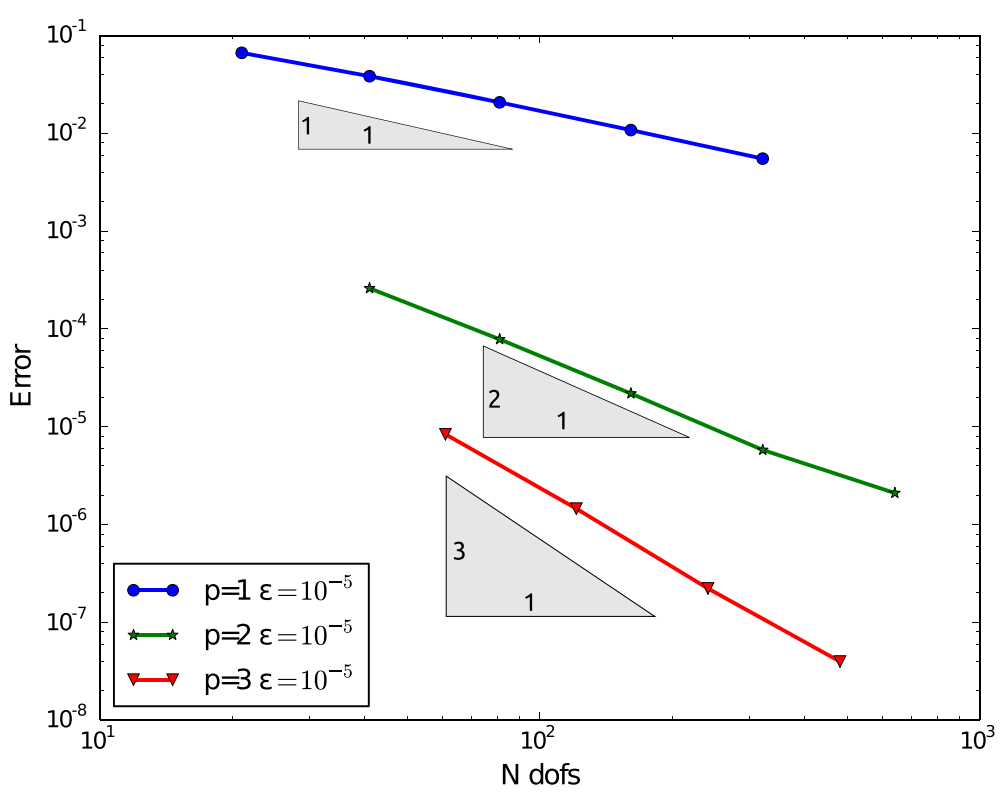

Figure $12 h$-convergence, enriched approximation.

seen in Figure 15 that, as expected, exponential convergences are obtained for $q=0.056$ and 0.24. The performances are similar for both meshes, although being less efficient than the enriched approximation. The small exponential convergence for $q=0.0032$ may seem surprising, as this value is out of the range from eqn. (28). In fact, condition (28) was obtained for an exponential convergence with quadratic polynomials. If one accepts to shift the exponential convergence to higher polynomial orders, smaller progressions

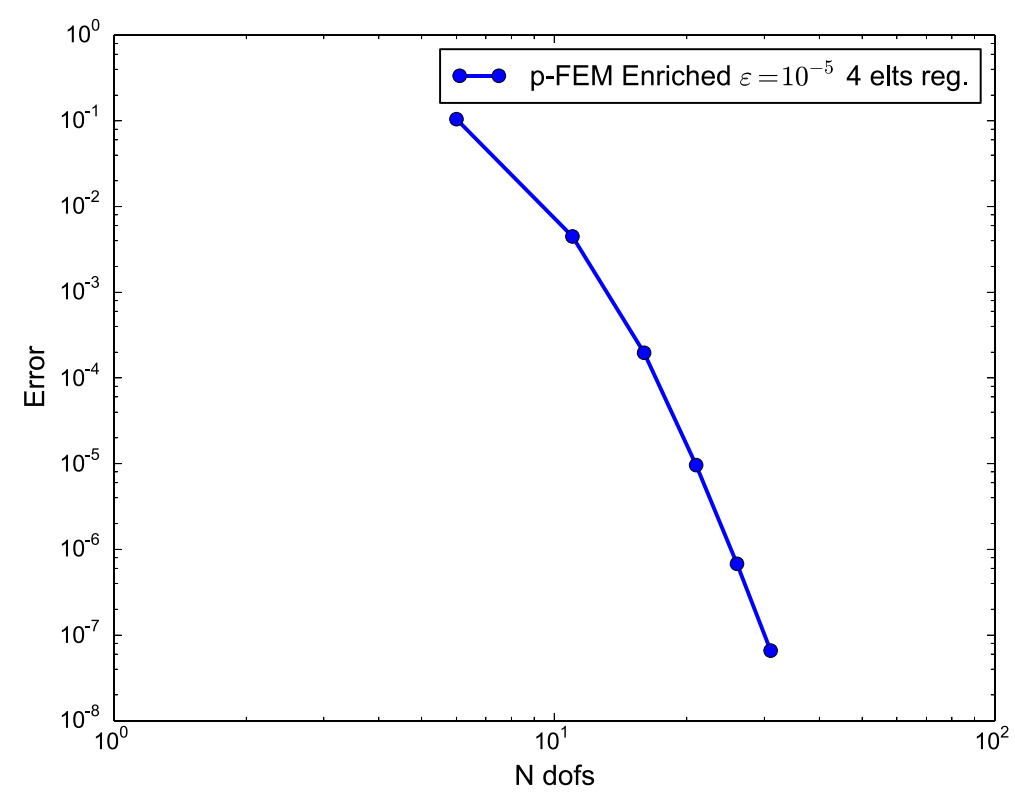

Figure $13 p$-convergence, enriched approximation. 


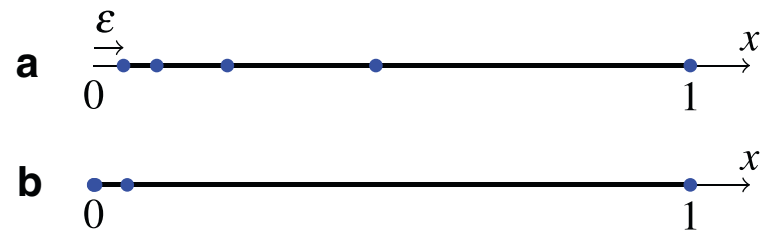

Figure 14 Unidimensional geometric mesh for 4 elements. a): illustrative scale; b): real scale.

can be considered. In particular, $q=0.0032$ is associated to an exponential convergence for a P5 approximation.

As a conclusion for this section, one can see that it is possible to recover regular rates of convergence in the case of nearly-singular solutions if one of the proposed strategies is used. The enrichment of the approximation seems to be the more versatile approach, as it can be applied for both $h$ and $p$ refinement. In addition, it has been demonstrated on the proposed example that it was performing more efficiently than the mesh-based approach. However, note that the geometrical mesh approach is less prone to conditioning and integration issues, and that is remains applicable even when the asymptotic behaviour is not precisely known.

\section{Extension to 2D}

We now discuss the extension to $2 \mathrm{D}$ of the proposed strategies.

\section{Enrichment of the approximation}

The adaptation is straightforward in this case. The vectorial formulation presented in eqn. (15) is considered, and a straight study of the asymptotic mechanical fields (13) can be

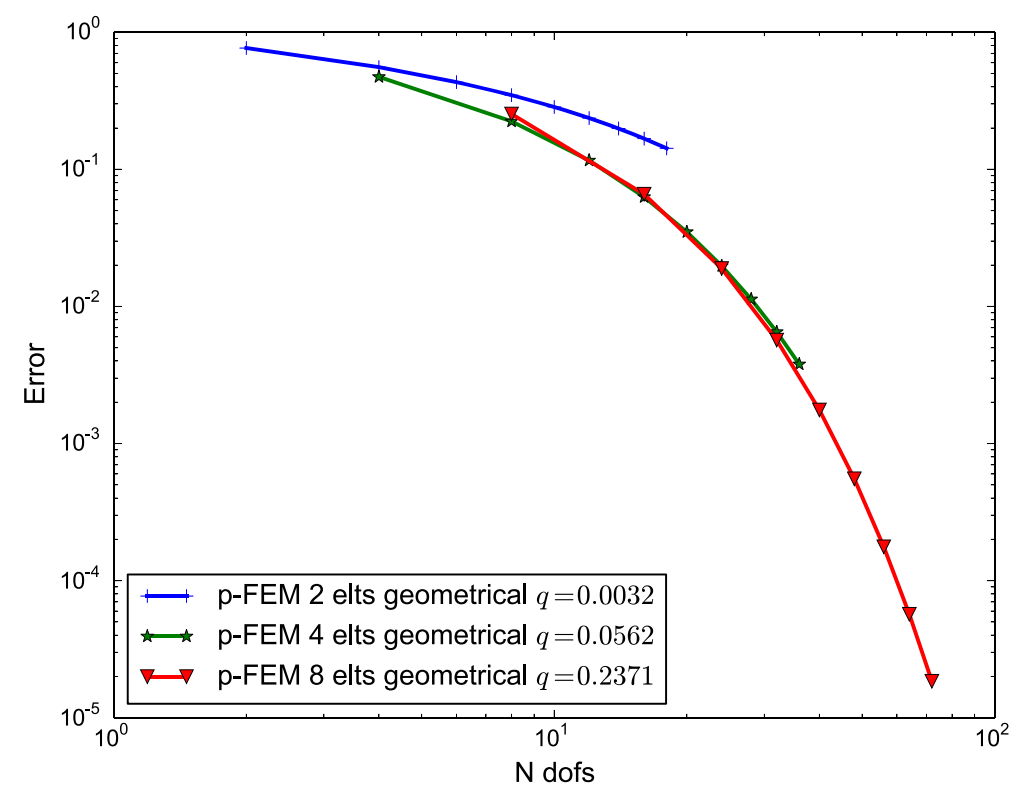

Figure $15 p$-convergence, geometrical meshes. 
used to build the enrichment functions. In this case, four vectorial enrichment functions $\varphi_{i}$ will be used:

$$
\begin{aligned}
& \varphi_{1}=u_{r}^{s, 1}(r, \theta) \mathbf{e}_{r}+u_{\theta}^{s, 1}(r, \theta) \mathbf{e}_{\theta} \\
& \boldsymbol{\varphi}_{2}=u_{r}^{s, 2}(r, \theta) \mathbf{e}_{r}+u_{\theta}^{s, 2}(r, \theta) \mathbf{e}_{\theta} \\
& \varphi_{3}=u_{r}^{b, 1}(r, \theta) \mathbf{e}_{r}+u_{\theta}^{b, 1}(r, \theta) \mathbf{e}_{\theta} \\
& \boldsymbol{\varphi}_{4}=u_{r}^{b, 2}(r, \theta) \mathbf{e}_{r}+u_{\theta}^{b, 2}(r, \theta) \mathbf{e}_{\theta}
\end{aligned}
$$

where $u_{r}^{s, i}$ and $u_{r}^{b, i}$ are given in equations (30)-(33).

\section{Geometrical mesh}

In the case of the construction of an adapted mesh, the objective is to be able to blend a geometrical mesh into an existing finite element grid. The implementation is easy as the computational mesh does not need to conform to the geometry, and follows the three steps depicted in Figure 16. (i) the element containing the center of the singularity is removed from the mesh together with its neighbours. (ii) a geometrical nodes patch is inserted in the vacant space (Figure 16b)). The geometrical progression of this patch is obtained taking into account to the requested number of elements made by the user, and prescribing the length of the smallest elements to $\rho$ (note that the value of the progression has a limited influence on the numerical efficiency, as shown in the following). Finally (iii), a local Delaunay algorithm is used in order to fill the space with finite elements, and build a transition to the existing elements (Figure 16c)). An example output of this procedure is given in Figure 17. Note that the center of the progression is not the center of the radius of curvature, but the point from which the singularity emanates (see section 'Rounded corner').

\section{D numerical examples}

Consider the model problem depicted in Figure 18. It represents a plane domain whose behaviour is assumed linear elastic and containing a re-entrant corner with a fillet of radius $\rho$. Young's modulus is assumed to have a unit value, and Poisson's ratio is 0.3 . Exact tractions whose expression are given in section 'Rounded corner' are applied on the boundaries of the domain (note that because of the geometrical approximations used to derive the solution, exact tractions have to be applied even on the free surface, see Figure 2). In the following, $\rho$ will range from 0.0625 to 0.1 , and both $h$ and

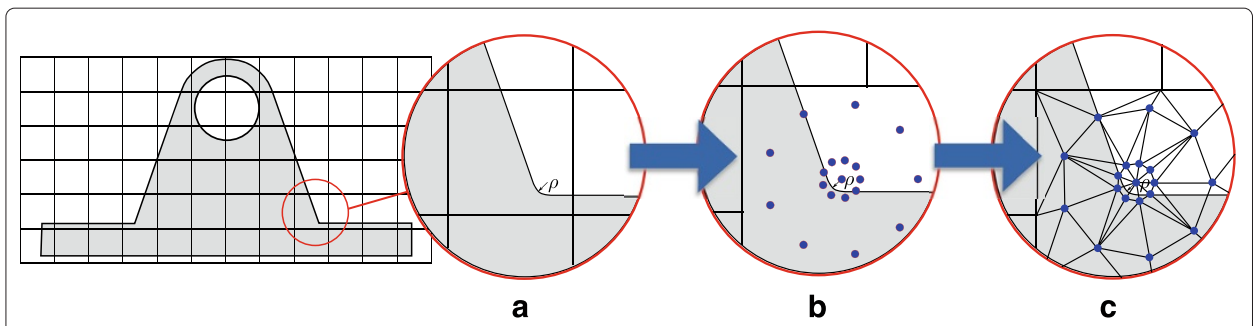

Figure 16 Blending of a geometrical mesh into an existing grid. a) Element containing the fillet; b) Removing neighbor cells and adding a cloud of nodes with a geometrical progression; c) Delaunay triangulation of the cavity. Note that the geometrical mesh is non-conforming. 


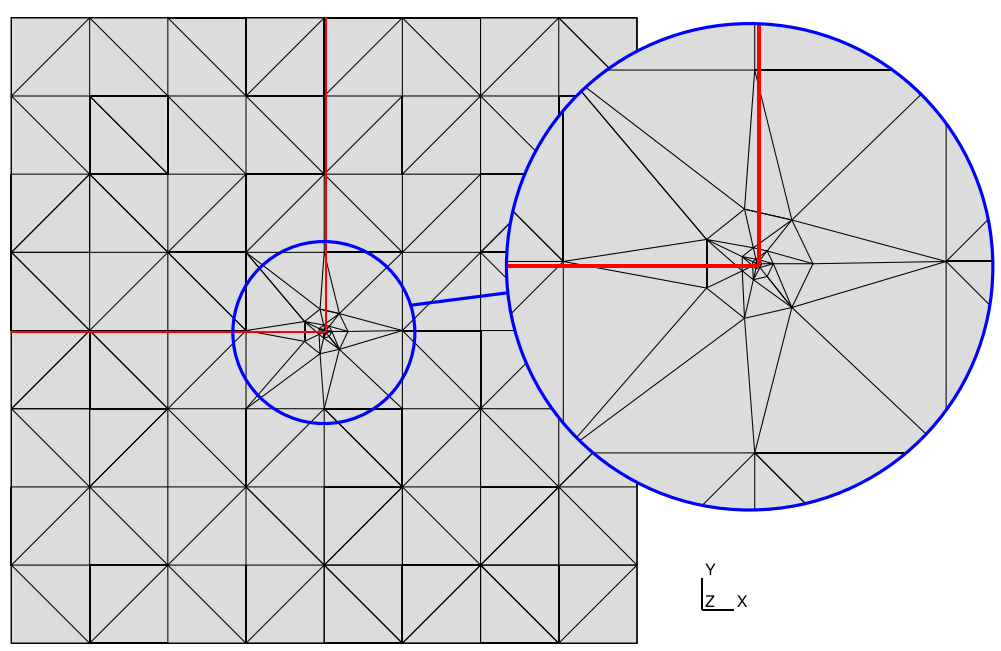

Figure 17 Resulting mesh for the L-shaped panel with a fillet (red: free-surface).

$p$-convergence are considered. In the former, only linear, quadratic and cubic approximations will be considered. In the case where an enrichment is considered, a "geometrical" enrichment strategy will be used by enriching all the elements lying in a circle of fixed radius centered on the center of the singularity. In order to integrate correctly the weak formulation in the enriched zone, the number of integration points is simply increased in the enriched elements, but not in the remaining part of the mesh. The objective of this section is to compare the performance of the $2 \mathrm{D}$ extension of the strategies considered in section 'Strategies for recovering optimal convergence', and propose good practice rules. All the numerical examples are conducted on a geometrical domain which consists in the bounding-box of the physical domain shown in Figure 18, and the X-FEM is used thanks to the definition of the geometry in terms of a level-set function. A sub-grid level-set $[6,7]$ approach is used in order to be able to represent the geometry accurately on coarse

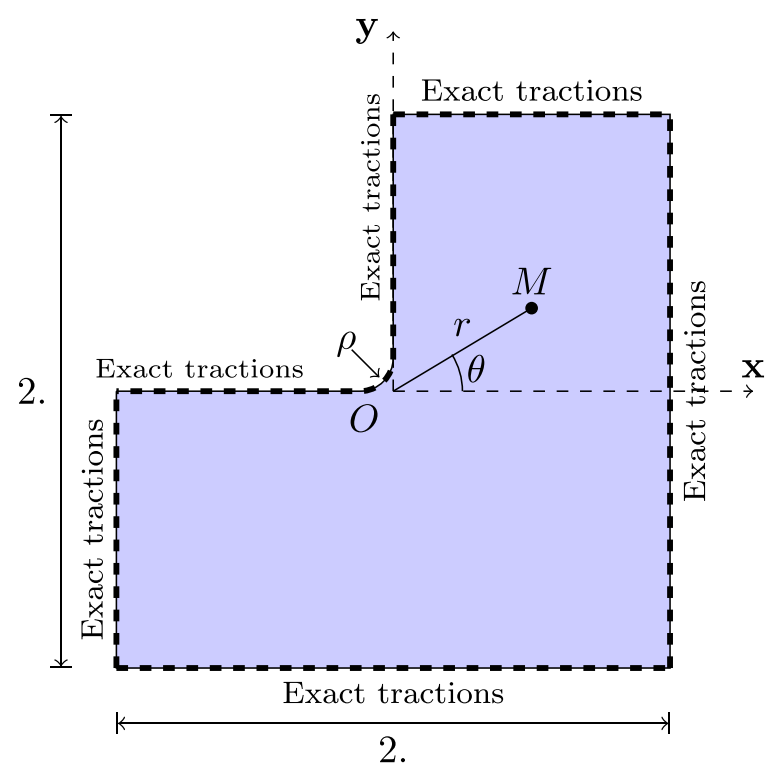

Figure 18 Blunt re-entrant corner convergence problem. 
computational meshes. Unless mentioned, the $h$-convergence studies are conducted on regular triangular meshes composed of $4 \times 4$ to $128 \times 128$ elements per side, and all errors correspond to relative errors.

\section{No enrichment}

In a first step, the case with no enrichment is considered with different values for $\rho$. The results obtained for $h$-convergence, and mode I are presented in Figure 19. The behaviour highlighted in the 1D model problem is also observed here: small $\rho$ prevent an $O\left(h^{p}\right)$ convergence, especially for low polynomial orders. It can be seen that the higher the polynomial order, the faster the convergence is recovered. The moment when this optimal convergence is recovered is now discussed: The convergence curves are now plotted with respect to $h / \rho$ rather than $h$ (see Figure 20). Looking at these curves, one can see that the change of regime in the convergence occurs in the shaded area. This means that the asymptotic convergence is recovered for $h \simeq \rho$ with linear elements. Moreover, this behaviour is consistent with the theoretical previsions from the last section (eqn. (25) for instance) Thus, the conclusion is the following: if no enrichment is used, one should consider meshes with element of size close to $\rho$ in the fillet area. This condition is more easily verified when conforming low order finite elements are used, as the construction of a conforming mesh already requires this amount of refinement for a proper geometrical representation. This is not the case with non-conforming methods, and $p$-Fem meshes using blending mapping as elements far bigger than the radius of the fillet can be considered. When quadratic or cubic approximations are used, the same behaviour occurs: the only difference comes from the fact that the optimal convergence is recovered faster than

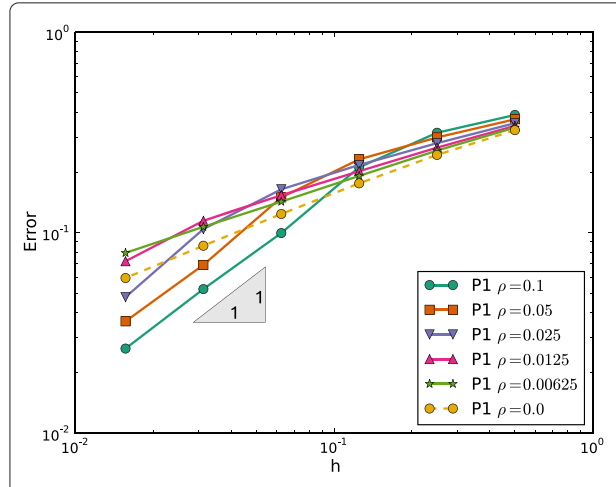

P1

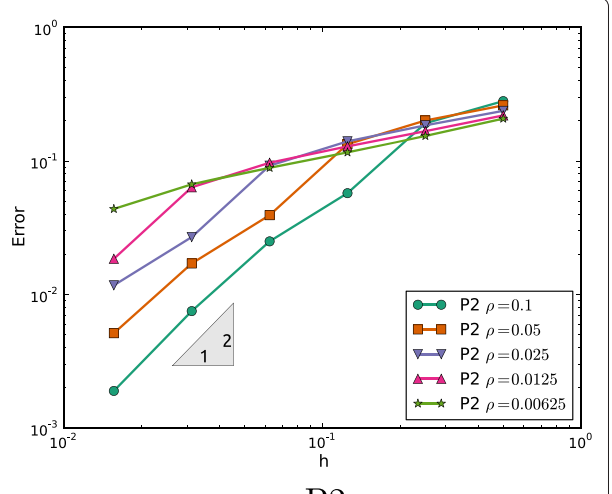

P2

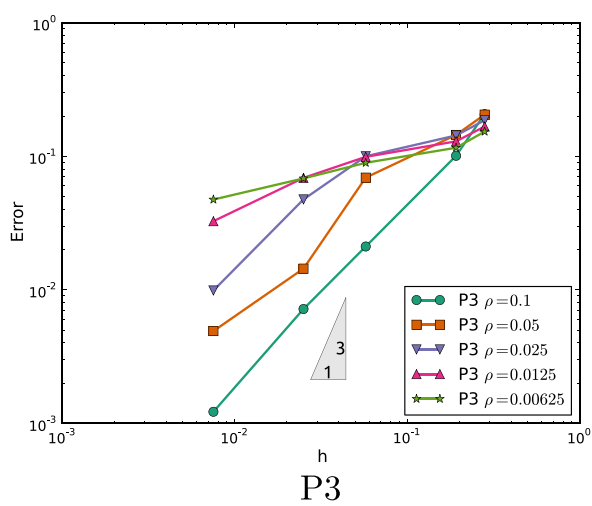

Figure $19 \mathrm{~h}$ convergence for linear, quadratic and cubic approximation with different fillet radius. 

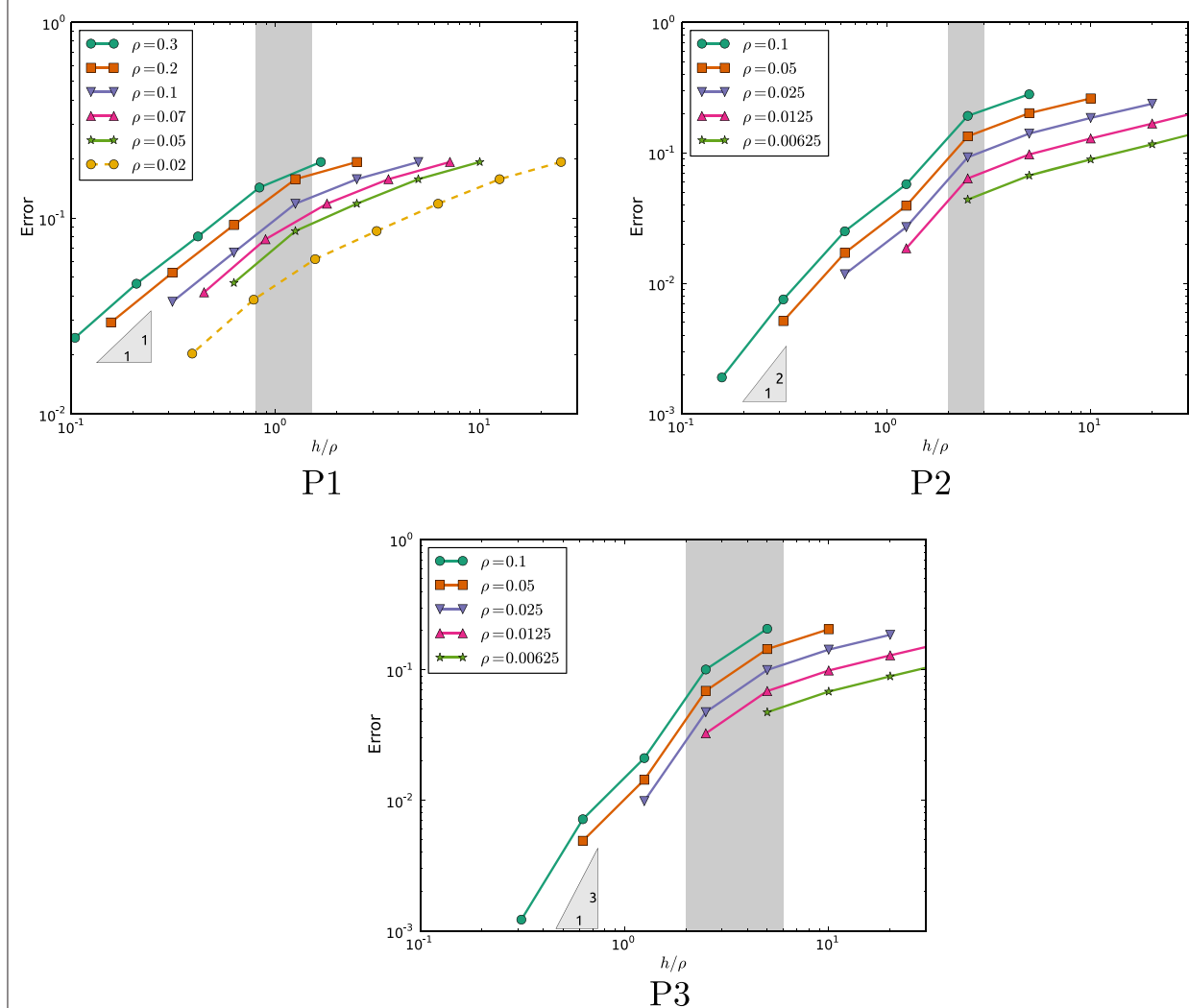

Figure 20 Convergence regime change with respect to $\rho$. The shaded zone represents the zone where the convergence regime is changing.

in the linear case. Yet, a very fine mesh has still to be considered ( $h \simeq 2-3 \times \rho$ for P2 and $h \simeq 3-6 \times \rho$ for P3). In addition, optimal convergence couldn't be recovered in our tests for small radii.

Finally, $p$-refinement is considered with $\rho=0.00625$ (smaller radius) in Figure 21. One can see that an algebraic rather than exponential convergence is obtained (as in the 1D case).

\section{Effect of the enrichment}

The approximation is now enriched by means of the enrichment functions presented in (29). The convergence study is done only for the smaller radius $(\rho=0.00625)$, mode 1 loading, and the enriched zone has a radius of 0.3. The results are presented in Figure 22. Regular convergence rates are recovered for linear, quadratic and cubic (not shown here) approximations, even for large element size.

Next, a $p$-convergence is performed on $16 \times 16$ and $8 \times 8$ meshes. The enriched zone is still a circular region of radius 0.30 and the results are given in Figure 23. Exponential convergence is recovered for both meshes with very accurate error levels $\left(10^{-4}\right)$ and moderate number of dofs. Note that if the desired accuracy can be obtained with a reasonable polynomial order (below P8), then the coarse mesh is more efficient. Otherwise, conditioning issues with higher polynomial orders may prevent to get a meaningful solution. In this case a finer mesh or a preconditioner such as [21] should be used. Another alternative could be to use a non-homogeneous $p$ distribution (smaller $p$ in the enriched area). 


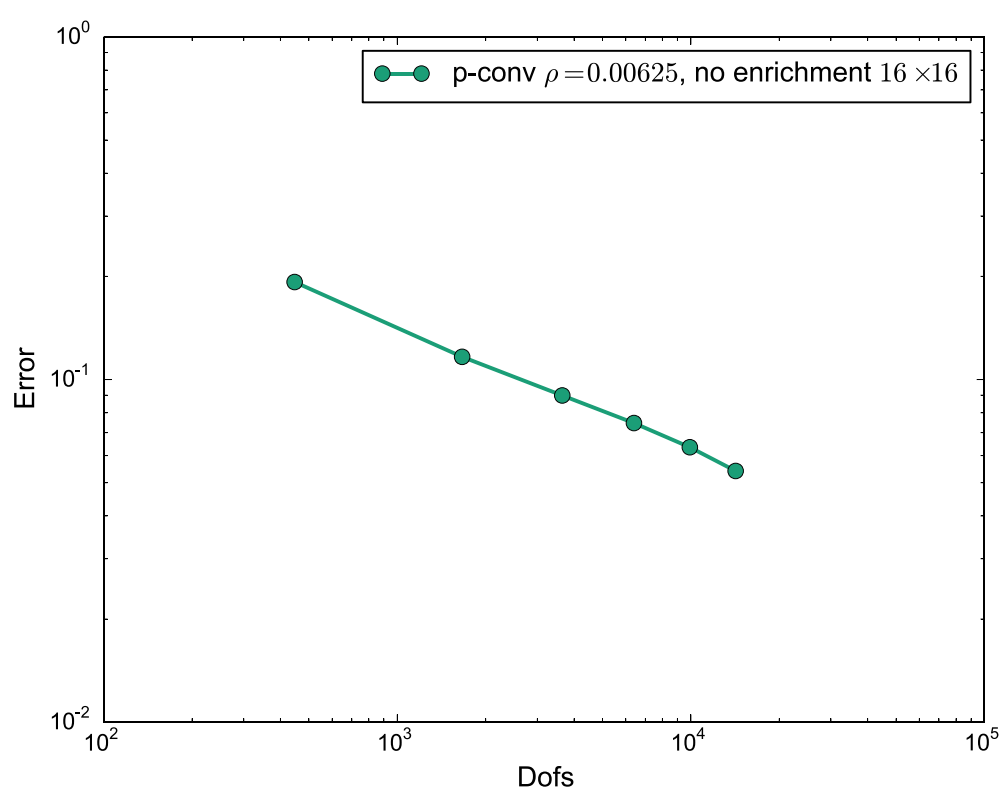

Figure $21 p$ convergence, no enrichment.

\section{Effect of mesh refinement}

Finally, the mesh-refinement strategy is evaluated. A $8 \times 8$ base mesh is considered (see Figure 17), and 4 or 5 layers of geometrical elements are focused near the center of the singularity (which is not the center of the radius of curvature). The results presented in Figure 24 show that an exponential convergence is obtained, and that the number of layers has very little influence on the performances of the approach.

\section{Comparison of the two strategies}

To conclude, the performances of the enrichment and geometrical mesh strategies are compared for $\rho=0.00625$. In the case of the use of an enrichment, the computational

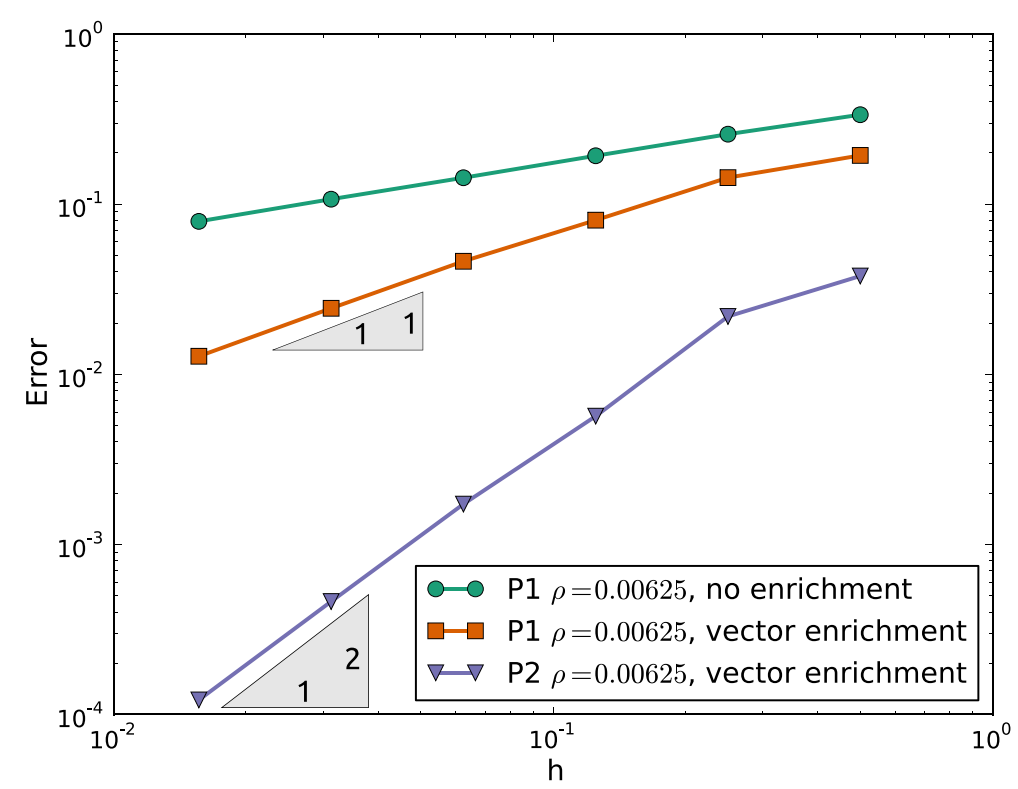

Figure $22 h$ convergence for linear, quadratic approximation with and without enrichment. 


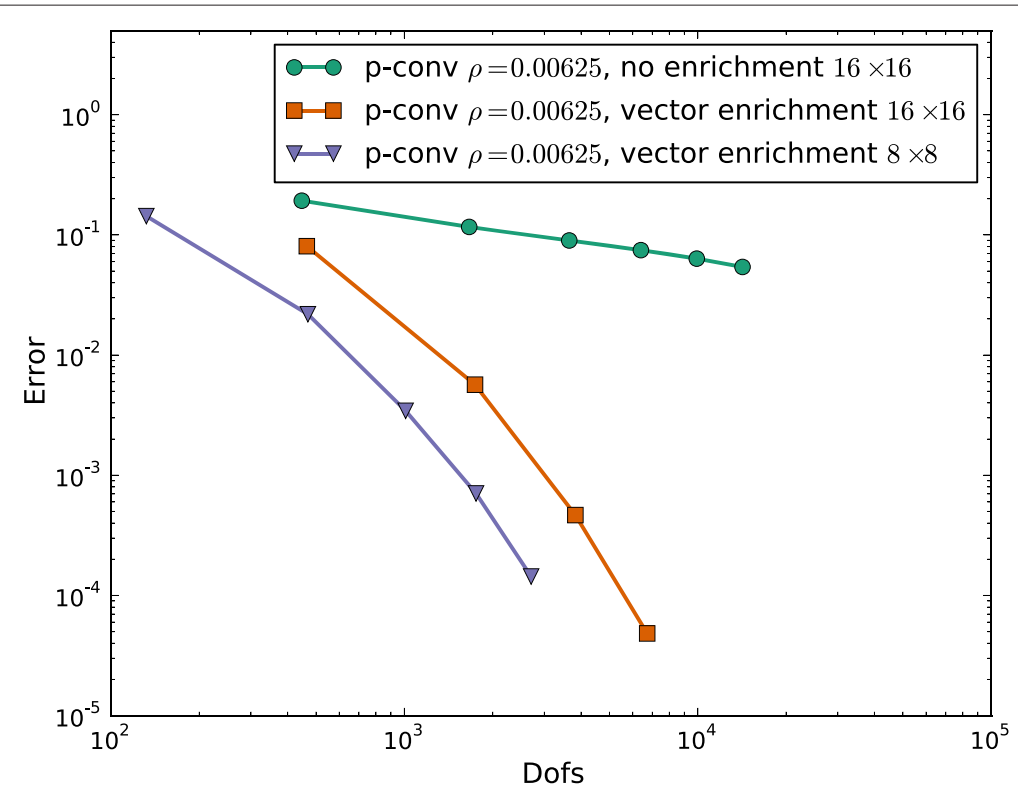

Figure $23 p$ convergence with and without enrichment, and using two different meshes.

setup is the same as in section 'Effect of the enrichment', whereas the number of layers is fixed for the geometrical mesh (but $4 \times 4$ and $8 \times 8$ base meshes are considered). The convergence curves are presented in Figure 25: it is shown that the behaviour of the different methods is quite similar, but that the enrichment strategy seems more efficient. However, it requires the derivation of the asymptotic fields for the problem of interest. Indeed, changing the boundary conditions on the free-surface of the corner has an influence on the order the singularity, and the trigonometric behaviour of the solution. On the contrary, the use of a geometrical mesh is more versatile.

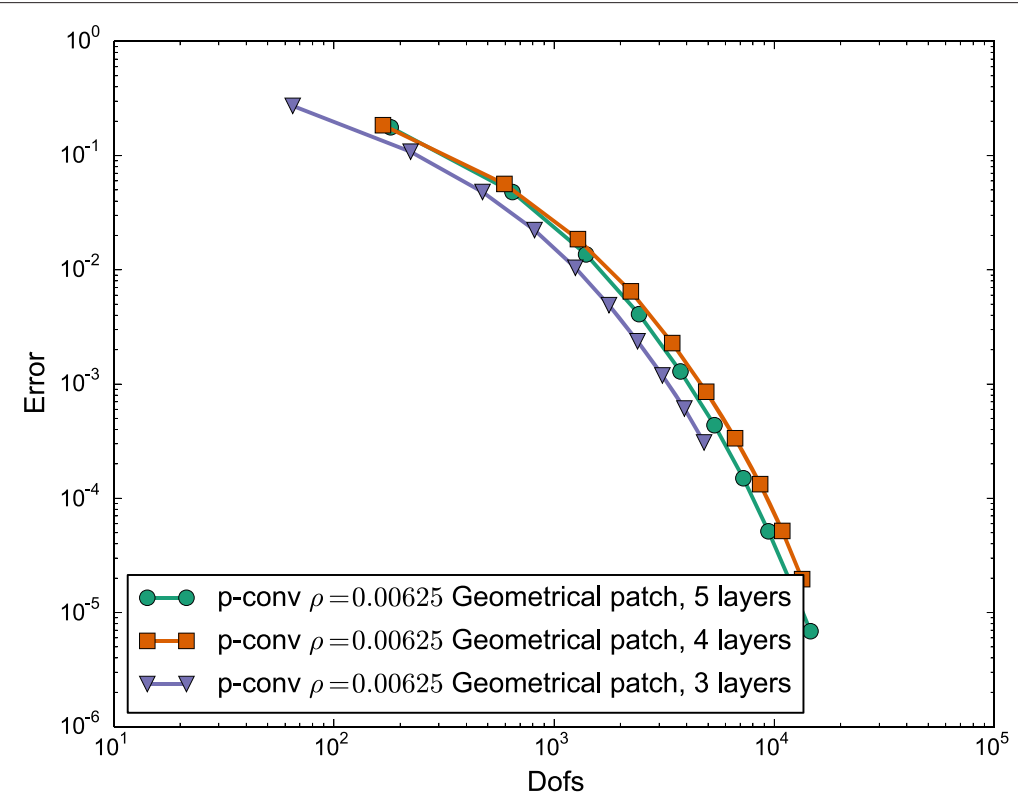

Figure $24 p$ convergence, geometrical mesh $(8 \times 8$ base mesh). 


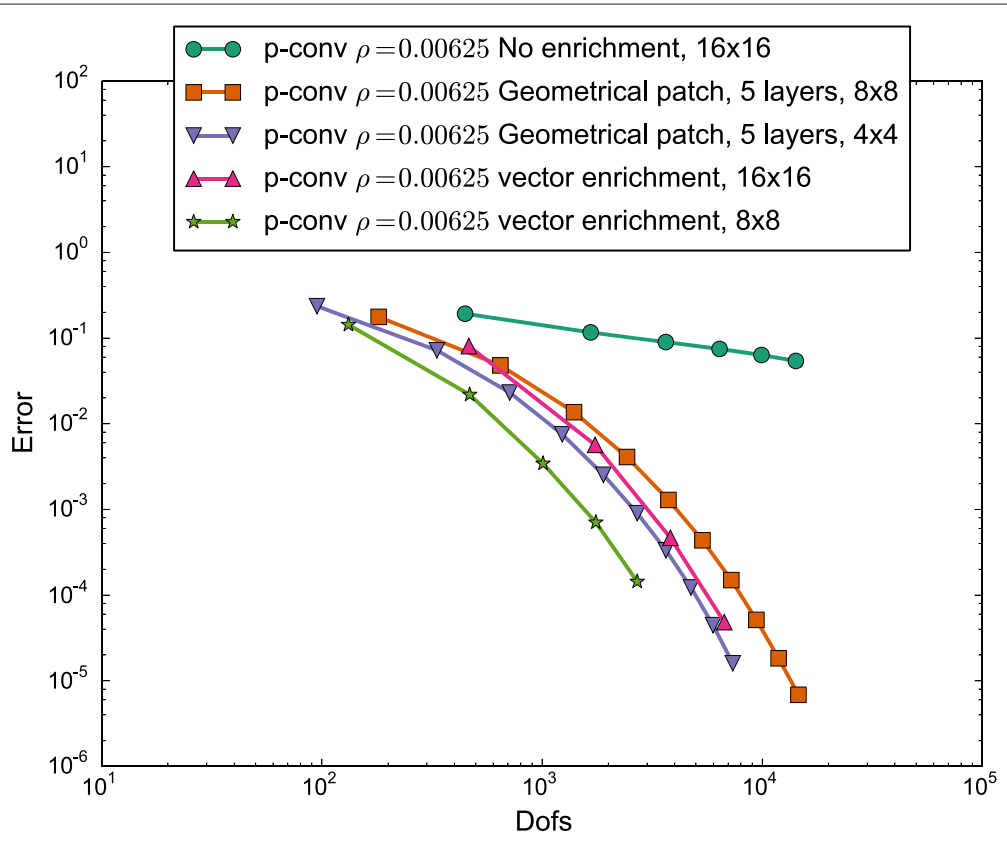

Figure 25 Comparison of two strategies, $p$ convergence.

\section{Conclusions}

In this contribution, the behaviour of non-conforming $h$ and $p$ finite elements has been studied in the case of nearly-singular solutions (re-entrant corners with a fillet here). In particular, it has been shown in both $1 \mathrm{D}$ and $2 \mathrm{D}$ that despite being regular, the convergence rate was algebraic and limited by the order of the singularity. Therefore, it is not possible to use fictitious domain methods such as the X-FEM without enrichment or the Finite-Cell Method if high accuracy is needed in near the fillet. Thanks to the study of a 1D model problem, it has been possible to highlight the reasons for such a behaviour. Two strategies have been proposed in order to overcome this convergence bound. The first one is based on the enrichment of the approximation near the fillet, and is usable for both $h$ and $p$ methods. The second one is based on the use of a mesh with a geometrical progression towards the center of the singularity, and is restricted to $p$ methods. The performances of these two strategies have been compared in both 1D and 2D: the enrichment method is the more efficient, but can lead to conditioning issues with highorder bases unless proper preconditioning strategies are used [21-24]. Moreover, the enrichment function is problem dependent, and can be tedious to obtain. In the present application, the enrichment functions are limited to stress-free bidimensional corners, and may be not be valid in the case where Dirichlet or non-homogeneous Neumann boundary conditions are applied. This kind of limitations was also present in the work of Wagner et al. [29] who considered rigid particles in Stokes flow, and used an enrichment function which is only valid for rigid and not too-close particles. The actual improvement with such not-fully adapted enrichment functions should be investigated further. On the contrary, exponential convergence is ensured in the case of the use of a geometrical mesh, no matter the progression, which makes it more versatile in case of high-order methods (only). The penalty of constructing such a mesh is greatly alleviated as this mesh does not need to conform the geometry, which is a contribution of the paper. 


\section{Endnotes}

${ }^{a}$ In (14), the vectorial nature of the field is handled by the shape functions, and not the dofs that are just coefficients. This notation facilitates the writing of the discrete operators.

${ }^{\mathrm{b}}$ For $\varepsilon=10^{-2}$ (Figure 9(b)), the maximum value of $r^{2}$ is 0.51 , which means that $\mathrm{p}$ convergence is obtained for the first element for any $p>2$.

\section{Appendix A: Asymptotic displacement fields}

The asymptotic displacement fields associated to the asymptotic stress fields (4), (5), (9) and (10) are obtained by proper integration of the strain equations. Their expressions are given below:

$$
\begin{aligned}
u_{r}^{s, i}= & \frac{r^{\lambda_{i}}}{2 G}\left[a_{1}\left(\kappa-\lambda_{i}\right) \cos \left[\left(\lambda_{i}-1\right) \theta\right]-a_{2}\left(\kappa-\lambda_{i}\right) \sin \left[\left(\lambda_{i}-1\right) \theta\right]\right. \\
& \left.-b_{1} \cos \left[\left(\lambda_{i}+1\right) \theta\right]+b_{2} \sin \left[\left(\lambda_{i}+1\right) \theta\right]\right] \\
u_{r}^{b, i}= & \frac{r^{\mu_{i}}}{2 G}\left[d_{1}\left(\kappa-\mu_{i}\right) \cos \left[\left(\mu_{i}-1\right) \theta\right]-d_{2}\left(\kappa-\mu_{i}\right) \sin \left[\left(\mu_{i}-1\right) \theta\right]\right. \\
& \left.-c_{1} \cos \left[\left(\mu_{i}+1\right) \theta\right]+c_{2} \sin \left[\left(\mu_{i}+1\right) \theta\right]\right] \\
u_{\theta}^{s, i}= & \frac{r^{\lambda_{i}}}{2 G}\left[a_{1}\left(\kappa+\lambda_{i}\right) \sin \left[\left(\lambda_{i}-1\right) \theta\right]+a_{2}\left(\kappa+\lambda_{i}\right) \cos \left[\left(\lambda_{i}-1\right) \theta\right]\right. \\
& \left.+b_{1} \sin \left[\left(\lambda_{i}+1\right) \theta\right]+b_{2} \cos \left[\left(\lambda_{i}+1\right) \theta\right]\right] \\
u_{\theta}^{b, i}= & \frac{r^{\mu_{i}}}{2 G}\left[d_{1}\left(\kappa+\mu_{i}\right) \sin \left[\left(\mu_{i}-1\right) \theta\right]+d_{2}\left(\kappa+\mu_{i}\right) \cos \left[\left(\mu_{i}-1\right) \theta\right]\right. \\
& +c_{1} \sin \left[\left(\mu_{i}+1\right) \theta\right]+c_{2} \cos \left[\left(\mu_{i}+1\right) \theta\right]
\end{aligned}
$$

The expression of $a_{1}, b_{1}, c_{1}$ as a function of $a_{1}$ and $b_{2}, c_{2}, d_{2}$ as a function of $a_{2}$ are also given in [14]:

$$
\begin{aligned}
& b_{1}=\chi_{b 1}\left(1-\lambda_{1}\right) a_{1} \\
& c_{1}=\frac{q \lambda_{1} r_{0}^{\lambda_{1}-\mu_{1}}}{4 \mu_{1}(q-1)} \chi_{c 1} a_{1} \\
& d_{1}=\frac{q \lambda_{1} r_{0}^{\lambda_{1}-\mu_{1}}}{4 \mu_{1}(q-1)} \chi_{d 1} a_{1} \\
& b_{2}=-\chi_{b 2}\left(1-\lambda_{2}\right) a_{2} \\
& c_{2}=\frac{q \lambda_{2} r_{0}^{\lambda_{2}-\mu_{2}}}{4 \mu_{2}\left(\mu_{2}-1\right)} \chi_{c 2} a_{2} \\
& d_{2}=\frac{q \lambda_{2} r_{0}^{\lambda_{2}-\mu_{2}}}{4 \mu_{2}\left(\mu_{2}-1\right)} \chi_{d 2} a_{2}
\end{aligned}
$$




\section{Authors' contributions}

GL worked on the algorithms, performed all the computations and drafted the manuscript. NM worked on the algorithms and carried out detailed revision. All authors read and approved the final manuscript.

\section{Acknowledgements}

The support of the ERC Advanced Grant XLS no 291102 is gratefully acknowledged

Received: 15 April 2014 Accepted: 26 June 2014

Published: 18 July 2014

\section{References}

1. Szabó B, Babuška I (1991) Finite element analysis. 1st edition. Wiley

2. Sevilla R, Fernández-méndez S (2008) NURBS-Enhanced Finite Element Method (NEFEM). Int J Numer Meth Eng 76:56-83

3. Sevilla R, Fernández-méndez S, Huerta S (2011) Comparison of high-order curved finite elements. Int J Numer Meth Eng 87:719-734

4. Moës N, Dolbow JE, Belytschko T (1999) A finite element method for crack growth without remeshing. Int J Numer Meth Eng 46:131-150

5. Saulèv VK (1963) On the solution of some boundary value problems on high performance computers by fictitious domain method. Siberian Math J 4:912-925

6. Dréau K, Chevaugeon N, Moës N (2010) Studied X-FEM enrichment to handle material interfaces with higher order finite element. Comput Meth Appl Mech Eng 199(29-32):1922-1936

7. Legrain G, Chevaugeon N, Dréau K (2012) High order X-FEM and levelsets for complex microstructures: uncoupling geometry and approximation. Comput Meth Appl Mech Eng 241-244:172-189

8. Groß S, Reusken A (2007) An extended pressure finite element space for two-phase incompressible flows with surface tension. J Comput Phys 224(1):40-58

9. Düster A, Rank E (2001) The p-version of the finite element method compared to an adaptive h-version for the deformation theory of plasticity. Comput Meth Appl Mech Eng 190:1925-1935

10. Legrain G (2013) A NURBS enhanced extended finite element approach for unfitted CAD analysis. Comput Mech 52(4):913-929

11. Melenk JM, Babuška I, Babuskab I (1996) The partition of unity finite element method: basic theory and applications. Comput Meth Appl Mech Eng 139:289-314

12. Williams ML (1952) Stress singularities resulting from various boundary conditions in angular corners of plates in tension. ASME J Appl Mech 19:526-528

13. Lazzarin $P$, Tovo R (1996) A unified approach to the evaluation of linear elastic stress fields in the neighborhood of cracks and notches. Int J Fract 78(1):3-19

14. Filippi S, Lazzarin P, Tovo R (2002) Developments of some explicit formulas useful to describe elastic stress fields ahead of notches in plates. Int J Solid Struct 39(17):4543-4565

15. Dini D, Hills DA (2004) Asymptotic characterisation of nearly-sharp notch root stress fields. Int J Fract 130(3):651-666

16. Creager M, Paris PC (1967) Elastic field equations for blunt cracks with reference to stress corrosion cracking. Int J Fract Mech 3(4):247-252

17. Glinka G (1985) Calculation of inelastic notch-tip strain- stress histories under cyclic loading. Eng Fract Mech 22(5):839-854

18. Sukumar N, Chopp DL, Moës N, Belytschko T (2001) Modeling holes and inclusions by level sets in the extended finite element method. Comp Meth Appl Mech Eng 190:6183-6200

19. Lian WD, Legrain G, Cartraud P (2012) Image-based computational homogenization and localization: comparison between X-FEM/levelset and voxel-based approaches. Comput Mech 51(3):279-293

20. Legrain G, Allais R, Cartraud P (2011) On the use of the extended finite element method with quadtree/octree meshes. Int J Numer Meth Eng 86:717-743

21. Béchet É, Minnebo H, Moës N, Burgardt B (2005) Improved implementation and robustness study of the X-FEM for stress analysis around cracks. Int J Numer Meth Eng 64(8):1033-1056

22. Laborde P, Pommier J, Renard Y, Salaün M (2005) High-order extended finite element method for cracked domains. Int J Numer Meth Eng 64(3):354-381

23. Babuška I, Banerjee U, Osborn JE (2004) Generalized finite element methods - main ideas, results and perspective. Int J Comput Methods 1(1):67-103

24. Ndeffo M, Massin P, Moës N (2013) Crack propagation modelisation using XFEM With 2D and 3D quadratic elements. In: International conference on Extended Finite Element Methods - XFEM 2013

25. Duarte CA, Babuška I, Oden JT (2000) Generalized finite element methods for three-dimensional structural mechanics problems. Comput Struct 77:215-232

26. Chevaugeon N, Moës N, Minnebo H (2013) Improved crack tip enrichment functions and integration for crack modeling using the extended finite element method. Int J Multiscale Comput Eng 11(6):597-631

27. Babuška I, Guo B (1992) The h, p and h-p version of the finite element method; basis theory and applications. Adv Eng Softw 15:159-174

28. Gui W, Babuška I (1986) The $p$ andh-p versions of the finite element method in 1 dimension Part l: the basic approximation results. Numerische Mathematik 612:577-612

29. Wagner GJ, Moës N, Liu WK, Belytschko T (2001) The extended finite element method for stokes flow past rigid cylinders. Int J Numer Meth Eng 51:293-313 